\title{
The Coulomb gauged vector potential formulation for the eddy-current problem in general geometry: well-posedness and numerical approximation
}

\author{
Oszkár BÍRÓ ${ }^{(*)}$ and Alberto VALLI ${ }^{(* *)}$ \\ (*) Institute for Fundamentals and Theory in Electrical Engineering, Graz University \\ of Technology, Kopernikusgasse 24, A-8010 Graz, Austria. \\ (**) Dipartimento di Matematica, Università di Trento, 38050 Povo (Trento), Italy.
}

\begin{abstract}
In this paper we prove that, in a general geometric situation, the Coulomb gauged vector potential formulation of the eddy-current problem for the time-harmonic Maxwell equations is well-posed, i.e., its solution exists and is unique. Moreover, a quasi-optimal error estimate for its finite element approximation with nodal elements is proved. To illustrate the performances of the finite element algorithm, some numerical results are also presented.
\end{abstract}

Keywords: eddy-currents, vector potentials, Coulomb gauge, well-posedness, finite element approximation.

\section{Introduction}

Let us consider a bounded connected open set $\Omega \subset \mathbf{R}^{3}$, with boundary $\partial \Omega$. The unit outward normal vector on $\partial \Omega$ will be denoted by $\mathbf{n}$. We assume that $\bar{\Omega}$ is split into two parts, $\bar{\Omega}=\overline{\Omega_{C}} \cup \overline{\Omega_{I}}$, where $\Omega_{C}$ (a non-homogeneous nonisotropic conductor) and $\Omega_{I}$ (a perfect insulator) are open disjoint subsets, such that $\overline{\Omega_{C}} \subset \Omega$. For the sake of simplicity, we also suppose that $\Omega_{I}$ is connected (the general case can be treated in a similar way, focusing on each connected component of $\Omega_{I}$, but some modifications are needed when the boundary of a connected component of $\Omega_{I}$ has empty intersection with $\partial \Omega$ ).

We denote by $\Gamma:=\partial \Omega_{I} \cap \partial \Omega_{C}$ the interface between the two subdomains; note that, in the present situation, $\partial \Omega_{C}=\Gamma$ and $\partial \Omega_{I}=\partial \Omega \cup \Gamma$. Moreover, we indicate by $\Gamma_{j}, j=1, \ldots, p_{\Gamma}$, the connected components of $\Gamma$, and by $(\partial \Omega)_{t}, t=$ $0,1, \ldots, p_{\partial \Omega}$, the connected components of $\partial \Omega$ (in particular, we have denoted by $(\partial \Omega)_{0}$ the external one).

In this paper we study the time-harmonic eddy-current problem, in which the displacement current term $\frac{\partial \mathcal{D}}{\partial t}$ is neglected, and the electric field $\mathcal{E}$, the magnetic field $\mathcal{H}$ and the applied current density $\mathcal{J}_{e}$ are of the form

$$
\begin{aligned}
\mathcal{E}(t, \mathbf{x}) & =\operatorname{Re}[\mathbf{E}(\mathbf{x}) \exp (i \omega t)] \\
\mathcal{H}(t, \mathbf{x}) & =\operatorname{Re}[\mathbf{H}(\mathbf{x}) \exp (i \omega t)] \\
\mathcal{J}_{e}(t, \mathbf{x}) & =\operatorname{Re}\left[\mathbf{J}_{e}(\mathbf{x}) \exp (i \omega t)\right]
\end{aligned}
$$

where $\omega \neq 0$ is a given angular frequency (see, e.g., Bossavit [7], p. 219). 
The constitutive relation $\mathcal{B}=\mu \mathcal{H}$ (where $\mu$ is the magnetic permeability coefficient) is assumed to hold, as well as the (generalized) Ohm's law $\mathcal{J}=$ $\sigma \mathcal{E}+\mathcal{J}_{e}$ (where $\sigma$ is the electric conductivity).

The magnetic permeability $\mu$ is assumed to be a symmetric matrix, uniformly positive definite in $\Omega$, with entries in $L^{\infty}(\Omega)$. Since $\Omega_{I}$ is a perfect insulator, we require that $\sigma_{\mid \Omega_{I}} \equiv 0$; moreover, as $\Omega_{C}$ is a non-homogeneous non-isotropic conductor, $\sigma_{\mid \Omega_{C}}$ is assumed to be a symmetric matrix, uniformly positive definite in $\Omega_{C}$, with entries in $L^{\infty}\left(\Omega_{C}\right)$. Moreover, the dielectric coefficient $\varepsilon_{I}$, which enters the problem when one has to determine the electric field in $\Omega_{I}$, is assumed to be a symmetric matrix, uniformly positive definite in $\Omega_{I}$, with entries in $L^{\infty}\left(\Omega_{I}\right)$. Finally, the applied current density $\mathcal{J}_{e}$ is not assumed to vanish in $\Omega_{C}$, so that also the skin effect in current driven massive conductors can be modelled.

Concerning the boundary condition, we will consider in the magnetic boundary value problem, modelling a cavity within an infinitely permeable iron: namely, $\mathbf{H} \times \mathbf{n}$, representing the tangential component of the magnetic field, is assumed to vanish on $\partial \Omega$. The case of the electric boundary value problem, in which $\mathbf{E} \times \mathbf{n}$, representing the tangential component of the electric field, is assumed to vanish on $\partial \Omega$, can be treated following a similar approach, but in the sequel we will not dwell on it (its general description can be found, e.g., in Alonso Rodríguez, Fernandes and Valli [3]).

We will make the following assumptions on the geometry of $\Omega$ :

either $\partial \Omega \in C^{1,1}$, or else $\Omega$ is a Lipschitz polyhedron; the same assumption holds for $\Omega_{C}$ and $\Omega_{I}$.

As a consequence, it can be proved (see e.g., Foias and Temam [15], Picard [20], Amrouche, Bernardi, Dauge and Girault [4], Fernandes and Gilardi [14], Hiptmair [17]) that the space of harmonic vector fields

$$
\begin{array}{r}
\mathcal{H}_{\mu_{I}}(\partial \Omega ; \Gamma):=\left\{\mathbf{v}_{I} \in\left(L^{2}\left(\Omega_{I}\right)\right)^{3} \mid \operatorname{rot} \mathbf{v}_{I}=\mathbf{0}, \operatorname{div}\left(\mu_{I} \mathbf{v}_{I}\right)=0,\right. \\
\left.\mathbf{v}_{I} \times \mathbf{n}=\mathbf{0} \text { on } \partial \Omega, \mu_{I} \mathbf{v}_{I} \cdot \mathbf{n}_{I}=0 \text { on } \Gamma\right\}
\end{array}
$$

has a finite dimension, larger than $p_{\partial \Omega}$, say $p_{\partial \Omega}+n_{\Gamma}$, and that the space of harmonic vector fields

$$
\begin{array}{r}
\mathcal{H}_{\varepsilon_{I}}(\Gamma ; \partial \Omega):=\left\{\mathbf{v}_{I} \in\left(L^{2}\left(\Omega_{I}\right)\right)^{3} \mid \operatorname{rot} \mathbf{v}_{I}=\mathbf{0}, \operatorname{div}\left(\varepsilon_{I} \mathbf{v}_{I}\right)=0\right. \\
\left.\mathbf{v}_{I} \times \mathbf{n}_{I}=\mathbf{0} \text { on } \Gamma, \varepsilon_{I} \mathbf{v}_{I} \cdot \mathbf{n}=0 \text { on } \partial \Omega\right\}
\end{array}
$$

has a finite dimension, larger than $p_{\Gamma}-1$, say $p_{\Gamma}-1+n_{\partial \Omega}$. Moreover

there exist $n_{\Gamma}$ "cuts" $\Xi_{l}$, which are the interior of two-dimensional, mutually disjoint, compact and connected Lipschitz manifolds $\overline{\Xi_{l}}$ with boundary $\partial \Xi_{l}$, such that $\Xi_{l} \subset \Omega_{I}$ and $\partial \Xi_{l} \subset \Gamma$, and such that in the open set $\widehat{\Omega_{I}}:=\Omega_{I} \backslash \cup_{l} \Xi_{l}$, assumed to be connected, every curl-free vector field with vanishing tangential component on $\partial \Omega$ has a global potential; 
there exist $n_{\partial \Omega}$ "cuts" $\Sigma_{k}$, which are the interior of two-dimensional, mutually disjoint, compact and connected Lipschitz manifolds $\overline{\Sigma_{k}}$ with boundary $\partial \Sigma_{k}$, such that $\Sigma_{k} \subset \Omega_{I}$ and $\partial \Sigma_{k} \subset \partial \Omega$, and such that in the open set $\widetilde{\Omega_{I}}:=\Omega_{I} \backslash \cup_{k} \Sigma_{k}$, assumed to be connected, every curl-free vector field with vanishing tangential component on $\Gamma$ has a global potential.

In particular, $n_{\Gamma}$ is the number of singular (or non-bounding) cycles in $\overline{\Omega_{I}}$ of first type, namely those cycles on $\Gamma$ that cannot be represented as $\partial S \backslash \boldsymbol{\gamma}, S$ being a surface contained in $\Omega_{I}$ and $\boldsymbol{\gamma}$ a cycle, possibly empty, contained in $\partial \Omega$. Similarly, $n_{\partial \Omega}$ is the number of singular cycles in $\overline{\Omega_{I}}$ of second type, namely, those cycles on $\partial \Omega$ that cannot be represented as $\partial S \backslash \boldsymbol{\gamma}, S$ being a surface contained in $\Omega_{I}$ and $\boldsymbol{\gamma}$ a cycle, possibly empty, contained in $\Gamma$.

Let us assume that the current density $\mathbf{J}_{e} \in\left(L^{2}(\Omega)\right)^{3}$ satisfies the (necessary) conditions

$$
\begin{gathered}
\operatorname{div} \mathbf{J}_{e, I}=0 \text { in } \Omega_{I} \quad, \quad \mathbf{J}_{e, I} \cdot \mathbf{n}=0 \text { on } \partial \Omega \\
\int_{\Gamma_{j}} \mathbf{J}_{e, I} \cdot \mathbf{n}_{I}=0 \quad \forall j=1, \ldots, p_{\Gamma}-1 \\
\int_{\Omega_{I}} \mathbf{J}_{e, I} \cdot \boldsymbol{\pi}_{k, I}=0 \quad \forall k=1, \ldots, n_{\partial \Omega},
\end{gathered}
$$

where $\boldsymbol{\pi}_{k, I}$ are basis functions of the space $\mathcal{H}_{\varepsilon_{I}}(\Gamma ; \partial \Omega)$ that are not gradients. As it will be shown in Section 4, condition $\left(\mathrm{H}_{2}\right)_{3}$ is equivalent to setting the total applied current through $\Sigma_{k}$ to zero. This is necessary in view of Ampère law, since $\mathbf{H} \times \mathbf{n}$ vanishes on $\partial \Omega$, hence on $\partial \Sigma_{k}$.

(For the ease of the reader, in $(H 2)$ and in the sequel we are always denoting the duality pairings as surface integrals; see Bossavit [7], Dautray and Lions [12], Girault and Raviart [16] for more details on these aspects related to functional analysis and to linear spaces of functions.)

In Alonso Rodríguez, Fernandes and Valli [3] it has been proved that the complete system of equations describing the eddy-current problem in terms of 
the magnetic field $\mathbf{H}$ and the electric field $\mathbf{E}_{C}$ is:

$$
\begin{cases}\operatorname{rot} \mathbf{E}_{C}+i \omega \mu_{C} \mathbf{H}_{C}=\mathbf{0} & \text { in } \Omega_{C} \\ \operatorname{rot} \mathbf{H}_{C}-\sigma \mathbf{E}_{C}=\mathbf{J}_{e, C} & \text { in } \Omega_{C} \\ \operatorname{rot} \mathbf{H}_{I}=\mathbf{J}_{e, I} & \text { in } \Omega_{I} \\ \operatorname{div}\left(\mu_{I} \mathbf{H}_{I}\right)=0 & \text { in } \Omega_{I} \\ \int_{(\partial \Omega)_{t}} \mu_{I} \mathbf{H}_{I} \cdot \mathbf{n}=0 & \forall t=1, \ldots, p_{\partial \Omega} \\ \int_{\Omega_{I}} i \omega \mu_{I} \mathbf{H}_{I} \cdot \boldsymbol{\rho}_{l, I}+\int_{\Gamma}\left(\mathbf{E}_{C} \times \mathbf{n}_{C}\right) \cdot \boldsymbol{\rho}_{l, I}= & \forall l=1, \ldots, n_{\Gamma} \\ \mathbf{H}_{I} \times \mathbf{n}=\mathbf{0} & \text { on } \partial \Omega \\ \mu_{I} \mathbf{H}_{I} \cdot \mathbf{n}_{I}+\mu_{C} \mathbf{H}_{C} \cdot \mathbf{n}_{C}=0 & \text { on } \Gamma \\ \mathbf{H}_{I} \times \mathbf{n}_{I}+\mathbf{H}_{C} \times \mathbf{n}_{C}=\mathbf{0} & \text { on } \Gamma,\end{cases}
$$

where $\mathbf{n}_{C}=-\mathbf{n}_{I}$ is the unit outward normal vector on $\partial \Omega_{C}=\Gamma$, we have set $\mathbf{E}_{C}:=\mathbf{E}_{\mid \Omega_{C}}$ (and similarly for $\Omega_{I}$ and any other restriction of function), and $\boldsymbol{\rho}_{l, I}$ are the basis functions of the space of harmonic fields $\mathcal{H}_{\mu_{I}}(\partial \Omega ; \Gamma)$ that are not gradients. In particular, in Alonso Rodríguez, Fernandes and Valli [3] it has been proved that, under the assumptions $(H 1)-(H 2)$, problem (1.3) has a unique solution.

In the following, we are going to consider problem (1.3) in terms of the Coulomb gauged vector potential formulation. We will prove that this formulation is well-posed, namely, there exists a solution for it and this solution is unique.

Let us note that the problem of uniqueness has been already considered and solved by many authors (see, e.g., Bíró and Preis [6] and the references therein), at least when the domains $\Omega$ and $\Omega_{C}$ have a simple geometry. Moreover, still for the case of simple geometry and assuming that the eddy-current problem has been solved in terms of the fields $\mathbf{E}_{C}$ and $\mathbf{H}$, Fernandes [13] has determined the correct gauging conditions for the existence and uniqueness of the vector magnetic potential $\mathbf{A}$ and the scalar electric potential $V_{C}$.

In this paper, instead, we mainly focus on the problem of existence of these potentials in general geometry, obtaining the uniqueness as a consequence of the positivity of the energy functional; moreover, we will derive from this result the stability and the convergence of the finite element approximation by nodal elements. To our knowledge, similar results have been proved only for the formulation using the modified vector potential $\mathbf{A}_{C}^{*}$ (equivalent to the electric field $\mathbf{E}_{C}$ ) in $\Omega_{C}$, combined with the scalar magnetic potential $\psi_{I}$ in $\Omega_{I}$ (see Tsukerman [21], Alonso and Valli [2]).

In the last Section we present some numerical results that illustrate the performances of the approximation algorithm based on nodal finite elements. It 
will be shown that the efficiency of the method is improved introducing in the finite element space some constraints that, though not needed at the discrete level, are indeed necessary for the well-posedness of the infinite dimensional problem.

\section{The $\left(\mathbf{A}_{C}, V_{C}\right)-\mathbf{A}_{I}$ formulation}

In this case we are looking for a magnetic vector potential $\mathbf{A}$ and a scalar electric potential $V_{C}$ such that

$$
\mathbf{E}_{C}=-i \omega \mathbf{A}_{C}-i \omega \operatorname{grad} V_{C}, \quad \mu_{C} \mathbf{H}_{C}=\operatorname{rot} \mathbf{A}_{C}, \quad \mu_{I} \mathbf{H}_{I}=\operatorname{rot} \mathbf{A}_{I} .
$$

In this way one has $\operatorname{rot} \mathbf{E}_{C}=-i \omega \operatorname{rot} \mathbf{A}_{C}=-i \omega \mu_{C} \mathbf{H}_{C}$, and therefore the Faraday equation in $\Omega_{C}$ is satisfied. Moreover, $\mu_{I} \mathbf{H}_{I}$ is a solenoidal field in $\Omega_{I}$ and has a vanishing flux through any closed surface in $\overline{\Omega_{I}}$.

The matching conditions for $\mu \mathbf{H} \cdot \mathbf{n}$ can be expressed as

$$
\mathbf{A}_{I} \times \mathbf{n}_{I}+\mathbf{A}_{C} \times \mathbf{n}_{C}=\mathbf{0} \text { on } \Gamma
$$

as taking the tangential divergence of this relation one finds

$$
\operatorname{rot} \mathbf{A}_{I} \cdot \mathbf{n}_{I}+\operatorname{rot} \mathbf{A}_{C} \cdot \mathbf{n}_{C}=0 \quad \text { on } \Gamma \text {. }
$$

As a consequence, we have $\mu \mathbf{H}=\operatorname{rot} \mathbf{A}$ in the whole $\Omega$.

In order to have a unique vector potential $\mathbf{A}$, it is necessary to impose some gauge conditions: here we are considering the Coulomb gauge $\operatorname{div} \mathbf{A}=0$ in $\Omega$, with $\mathbf{A} \cdot \mathbf{n}=0$ on $\partial \Omega$. Therefore, we are also implicitly requiring the additional matching condition $\mathbf{A}_{I} \cdot \mathbf{n}_{I}+\mathbf{A}_{C} \cdot \mathbf{n}_{C}=0$ on $\Gamma$.

In a general geometric situation, this can be not enough for determining a unique vector potential $\mathbf{A}$ in $\Omega$. In fact, there exist non-trivial irrotational, solenoidal and tangential vector fields, namely, the elements of the finite dimensional space of harmonic fields

$$
\mathcal{H}(m ; \Omega):=\left\{\mathbf{v} \in\left(L^{2}(\Omega)\right)^{3} \mid \operatorname{rot} \mathbf{v}=\mathbf{0}, \operatorname{div} \mathbf{v}=0, \mathbf{v} \cdot \mathbf{n}=0 \text { on } \partial \Omega\right\} .
$$

Let us denote by $n_{\partial \Omega}^{*}$ the dimension of this vector space. As before, it can be proved that

there exist $n_{\partial \Omega}^{*}$ "cuts" $\Sigma_{z}^{*}$, which are the interior of two-dimensional, mutually disjoint, compact and connected Lipschitz manifolds $\overline{\Sigma_{z}^{*}}$

with boundary $\partial \Sigma_{z}^{*}$, such that $\Sigma_{z}^{*} \subset \Omega$ and $\partial \Sigma_{z}^{*} \subset \partial \Omega$, and such that in the open set $\widetilde{\Omega}:=\Omega \backslash \cup_{z} \Sigma_{z}^{*}$, assumed to be connected, every curl-free vector has a global potential.

In other words, each surface $\Sigma_{z}^{*}, z=1, \ldots, n_{\partial \Omega}^{*}$, "cuts" a cycle on $\partial \Omega$ that is not bounding a surface contained in $\Omega$. 
In this context, we are in a position to make precise the additional conditions that we are going to require. Coming back to the family of $n_{\partial \Omega}$ "cuts" $\Sigma_{k}$ introduced in (1.2), we assume that

the family of "cuts" $\Sigma_{z}^{*}$ is coincident with

the family of "cuts" $\Sigma_{k}$ for $z, k=1, \ldots, n_{\partial \Omega}$

(in particular, $\Sigma_{z}^{*} \subset \Omega_{I}$ for each $z=1, \ldots, n_{\partial \Omega}$ ), whereas $\Sigma_{z}^{*} \cap \Omega_{C} \neq \emptyset$ for each $z=n_{\partial \Omega}+1, \ldots, n_{\partial \Omega}^{*}$.

As a consequence, we have $n_{\partial \Omega} \leq n_{\partial \Omega}^{*}$.

When $n_{\partial \Omega}=n_{\partial \Omega}^{*}$, this is telling us that we can choose the "cuts" in $\Omega$ associated to the vector space $\mathcal{H}(m ; \Omega)$ without intersecting $\Omega_{C}$. Conversely, when $n_{\partial \Omega}<n_{\partial \Omega}^{*}$ some of the "cuts" have to intersect the conductor $\Omega_{C}$ (for example, this happens when $\Omega$ and $\Omega_{C}$ are two coaxial tori, for which $n_{\partial \Omega}=0$ and $\left.n_{\partial \Omega}^{*}=1\right)$.

We are going to prove that the number of the needed additional conditions to be imposed to the vector field $\mathbf{A}$ is $n_{\partial \Omega}$. For a while, let us describe these conditions in the abstract form

$$
\mathcal{G}_{k}(\mathbf{A})=0 \quad \forall k=1, \ldots, n_{\partial \Omega},
$$

where $\mathcal{G}_{z}(\cdot), z=1, \ldots, n_{\partial \Omega}^{*}$, are a suitable set of linear functionals that we will make precise in the sequel (see (4.9) and (4.10)).

Since we would like to find a unique solution $\left(\mathbf{A}_{C}, V_{C}\right)-\mathbf{A}_{I}$, we have also to impose a suitable condition to $V_{C}$, for instance

$$
\int_{\Omega_{C, j}} V_{C \mid \Omega_{C, j}}=0,
$$

where $\Omega_{C, j}, j=1, \ldots, p_{\Gamma}$, are the connected components of $\Omega_{C}$. 
In conclusion, taking into account (1.3), we are left with the problem

$(2.4) \begin{cases}\operatorname{rot}\left(\mu_{C}^{-1} \operatorname{rot} \mathbf{A}_{C}\right)+i \omega \sigma \mathbf{A}_{C}+i \omega \sigma \operatorname{grad} V_{C}=\mathbf{J}_{e, C} & \text { in } \Omega_{C} \\ \operatorname{rot}\left(\mu_{I}^{-1} \operatorname{rot} \mathbf{A}_{I}\right)=\mathbf{J}_{e, I} & \text { in } \Omega_{I} \\ \int_{\Omega_{C, j}} V_{C \mid \Omega_{C, j}}=0 & \forall j=1, \ldots, p_{\Gamma} \\ \operatorname{div} \mathbf{A}_{C}=0 & \text { in } \Omega_{C} \\ \operatorname{div} \mathbf{A}_{I}=0 & \text { in } \Omega_{I} \\ \mathcal{G}_{k}(\mathbf{A})=0 & \forall k=1, \ldots, n_{\partial \Omega} \\ \mathbf{A}_{I} \cdot \mathbf{n}=0 & \text { on } \partial \Omega \\ \left(\mu_{I}^{-1} \operatorname{rot} \mathbf{A}_{I}\right) \times \mathbf{n}=\mathbf{0} & \text { on } \partial \Omega \\ \mathbf{A}_{I} \cdot \mathbf{n}_{I}+\mathbf{A}_{C} \cdot \mathbf{n}_{C}=0 & \text { on } \Gamma \\ \mathbf{A}_{I} \times \mathbf{n}_{I}+\mathbf{A}_{C} \times \mathbf{n}_{C}=\mathbf{0} & \text { on } \Gamma \\ \left(\mu_{I}^{-1} \operatorname{rot} \mathbf{A}_{I}\right) \times \mathbf{n}_{I}+\left(\mu_{C}^{-1} \operatorname{rot} \mathbf{A}_{C}\right) \times \mathbf{n}_{C}=\mathbf{0} & \text { on } \Gamma .\end{cases}$

As it is well known (see, e.g., Morisue [19]), the Coulomb gauge condition $\operatorname{div} \mathbf{A}=0$ in $\Omega$ can be incorporated in the Ampère equation. Introducing the constant $\mu_{*}>0$, representing a suitable average in $\Omega$ of the entries of the matrix $\mu$, one considers

$$
\begin{cases}\operatorname{rot}\left(\mu_{C}^{-1} \operatorname{rot} \mathbf{A}_{C}\right)-\mu_{*}^{-1} \operatorname{grad} \operatorname{div} \mathbf{A}_{C}+i \omega \sigma \mathbf{A}_{C}+i \omega \sigma \operatorname{grad} V_{C}=\mathbf{J}_{e, C} & \text { in } \Omega_{C} \\ \operatorname{rot}\left(\mu_{I}^{-1} \operatorname{rot} \mathbf{A}_{I}\right)-\mu_{*}^{-1} \operatorname{grad} \operatorname{div} \mathbf{A}_{I}=\mathbf{J}_{e, I} & \text { in } \Omega_{I} \\ \operatorname{div}\left(i \omega \sigma \mathbf{A}_{C}+i \omega \sigma \operatorname{grad} V_{C}\right)=\operatorname{div} \mathbf{J}_{e, C} & \text { in } \Omega_{C} \\ \left(i \omega \sigma \mathbf{A}_{C}+i \omega \sigma \operatorname{grad} V_{C}\right) \cdot \mathbf{n}_{C}=\mathbf{J}_{e, C} \cdot \mathbf{n}_{C}+\mathbf{J}_{e, I} \cdot \mathbf{n}_{I} & \text { on } \Gamma,\end{cases}
$$

the last two conditions being necessary as the modification in the first two does not assure now that the electric field $\mathbf{E}_{C}=-i \omega \mathbf{A}_{C}-i \omega \operatorname{grad} V_{C}$ satisfies the necessary conditions $\operatorname{div}\left(\sigma \mathbf{E}_{C}\right)=-\operatorname{div} \mathbf{J}_{e, C}$ in $\Omega_{C}$ and $\sigma \mathbf{E}_{C} \cdot \mathbf{n}_{C}=-\mathbf{J}_{e, C} \cdot \mathbf{n}_{C}-$ $\mathbf{J}_{e, I} \cdot \mathbf{n}_{I}$ on $\Gamma$.

The complete $\left(\mathbf{A}_{C}, V_{C}\right)-\mathbf{A}_{I}$ formulation that we are going to consider is 
therefore

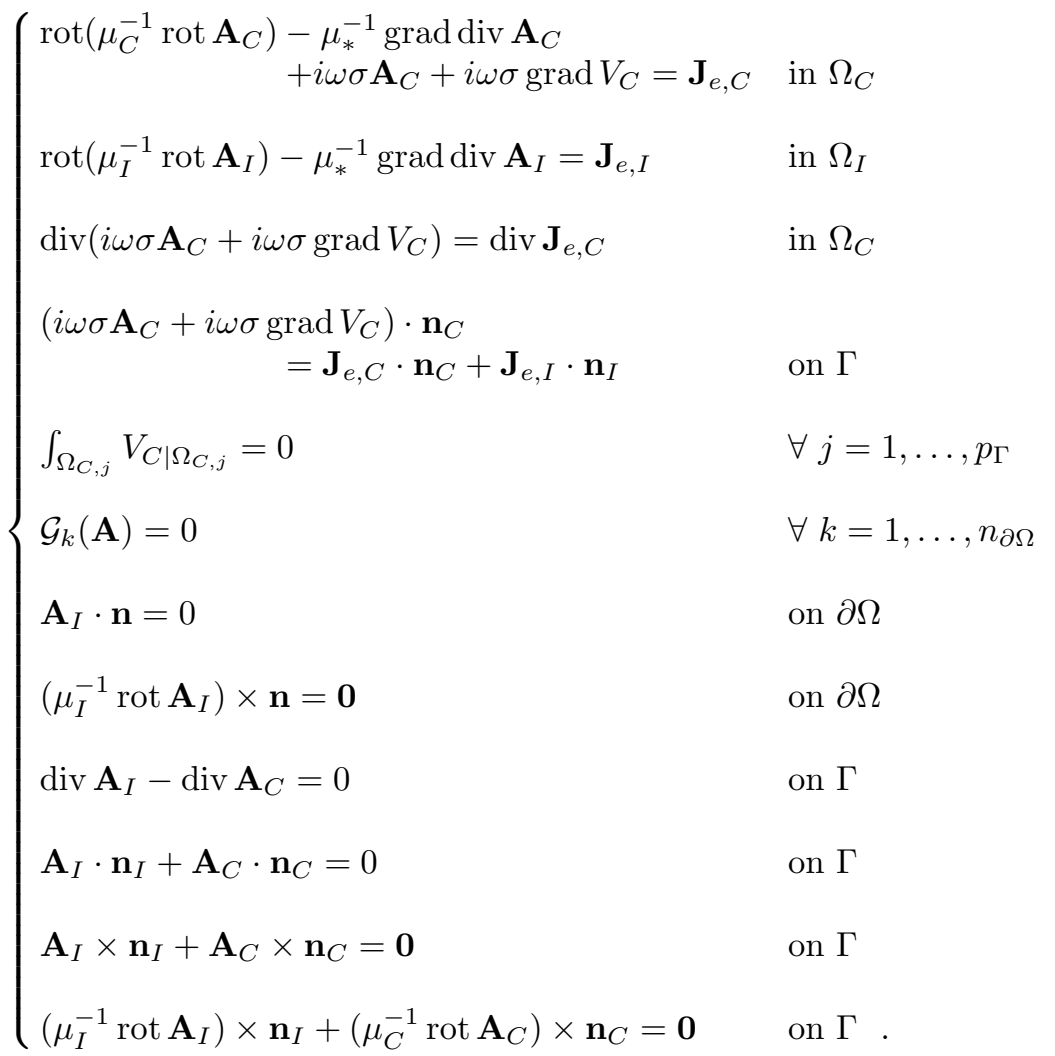

The interface condition $\operatorname{div} \mathbf{A}_{I}=\operatorname{div} \mathbf{A}_{C}$ on $\Gamma$ is necessary to assure that, for a solution to (2.5), one has $\operatorname{div} \mathbf{A}=0$ in the whole $\Omega$, and therefore one can recover from (2.5) the original system (2.4). In fact, we can prove

Lemma 2.1. For any solution $\left(\mathbf{A}_{C}, V_{C}\right)-\mathbf{A}_{I}$ to (2.5) one has $\operatorname{div} \mathbf{A}=0$ in $\Omega$, therefore $\left(\mathbf{A}_{C}, V_{C}\right)-\mathbf{A}_{I}$ is indeed a solution to $(2.4)$.

Proof. First of all we see that, from $(2.5)_{10}$, one sees that the divergence of $\mathbf{A}$ is well defined in the whole $\Omega$, and it holds $\operatorname{div} \mathbf{A}=\operatorname{div} \mathbf{A}_{I}$ in $\Omega_{I}$ and $\operatorname{div} \mathbf{A}=$ $\operatorname{div} \mathbf{A}_{C}$ in $\Omega_{C}$. Moreover, taking the divergence of the $(2.5)_{1}$ and using $(2.5)_{3}$ we have $-\Delta \operatorname{div} \mathbf{A}_{C}=0$ in $\Omega_{C}$. Recalling that the current density $\mathbf{J}$ satisfies by assumption $\operatorname{div} \mathbf{J}_{e, I}=0$ in $\Omega_{I}$, from $(2.5)_{2}$ one obtains also $-\Delta \operatorname{div} \mathbf{A}_{I}=0$ in $\Omega_{I}$. On the other hand, using $(2.5)_{4}$, on the interface $\Gamma$ we have

$$
\begin{aligned}
-\mu_{*}^{-1} \operatorname{grad} \operatorname{div} \mathbf{A}_{C} \cdot \mathbf{n}_{C} & =-\mathbf{J}_{e, I} \cdot \mathbf{n}_{I}-\operatorname{rot}\left(\mu_{C}^{-1} \operatorname{rot} \mathbf{A}_{C}\right) \cdot \mathbf{n}_{C} \\
& =-\mathbf{J}_{e, I} \cdot \mathbf{n}_{I}-\operatorname{div}_{\tau}\left[\left(\mu_{C}^{-1} \operatorname{rot} \mathbf{A}_{C}\right) \times \mathbf{n}_{C}\right],
\end{aligned}
$$

and also

$$
\begin{aligned}
-\mu_{*}^{-1} \operatorname{grad} \operatorname{div} \mathbf{A}_{I} \cdot \mathbf{n}_{I} & =\mathbf{J}_{e, I} \cdot \mathbf{n}_{I}-\operatorname{rot}\left(\mu_{I}^{-1} \operatorname{rot} \mathbf{A}_{I}\right) \cdot \mathbf{n}_{I} \\
& =\mathbf{J}_{e, I} \cdot \mathbf{n}_{I}-\operatorname{div}_{\tau}\left[\left(\mu_{I}^{-1} \operatorname{rot} \mathbf{A}_{I}\right) \times \mathbf{n}_{I}\right],
\end{aligned}
$$


therefore from $(2.5)_{12}$

$$
\operatorname{grad} \operatorname{div} \mathbf{A}_{C} \cdot \mathbf{n}_{C}+\operatorname{grad} \operatorname{div} \mathbf{A}_{I} \cdot \mathbf{n}_{I}=0 \quad \text { on } \Gamma .
$$

This last condition, together with the interface condition $(2.5)_{9}$, furnishes that $\operatorname{div} \mathbf{A}$ is a harmonic function in the whole $\Omega$. Moreover, using $(2.5)_{8}$, on the boundary $\partial \Omega$ we have

$$
\begin{aligned}
-\mu_{*}^{-1} \operatorname{grad} \operatorname{div} \mathbf{A} \cdot \mathbf{n} & =\mathbf{J}_{e, I} \cdot \mathbf{n}-\operatorname{rot}\left(\mu_{I}^{-1} \operatorname{rot} \mathbf{A}_{I}\right) \cdot \mathbf{n} \\
& =\mathbf{J}_{e, I} \cdot \mathbf{n}-\operatorname{div}_{\tau}\left[\left(\mu_{I}^{-1} \operatorname{rot} \mathbf{A}_{I}\right) \times \mathbf{n}\right]=0,
\end{aligned}
$$

as the current density satisfies by assumption $\mathbf{J}_{e, I} \cdot \mathbf{n}=0$ on $\partial \Omega$. As a consequence, we find that $\operatorname{div} \mathbf{A}$ is a constant in $\Omega$, and this constant must be 0 by $(2.5)_{7}$.

Note that, if $\left(\mathbf{A}_{C}, V_{C}\right)-\mathbf{A}_{I}$ is any solution of problem (2.4), then the corresponding $\mathbf{H}_{I}$ and $\mathbf{E}_{C}$ obtained through (2.1) satisfy

$$
\int_{\Omega_{I}} i \omega \mu_{I} \mathbf{H}_{I} \cdot \boldsymbol{\rho}_{l, I}+\int_{\Gamma}\left(\mathbf{E}_{C} \times \mathbf{n}_{C}\right) \cdot \boldsymbol{\rho}_{l, I}=0 \quad \forall l=1, \ldots, n_{\Gamma},
$$

as proved in Alonso Rodríguez, Fernandes and Valli [3]. Therefore, any solution to (2.4) gives, through (2.1), the solution to the eddy-current problem (1.3).

\section{The $\left(\mathbf{A}_{C}, V_{C}\right)-\mathbf{A}_{I}$ weak formulation}

We are now interested in finding a suitable weak formulation of (2.5). Taking a test function $\mathbf{v} \in H(\operatorname{rot} ; \Omega) \cap H(\operatorname{div} ; \Omega)$ with $\mathbf{v} \cdot \mathbf{n}=0$ on $\partial \Omega$, multiplying $(2.5)_{1}$ by $\overline{\mathbf{v}_{C}}$ (the complex conjugate of $\left.\mathbf{v}_{C}\right),(2.5)_{2}$ by $\overline{\mathbf{v}_{I}}$ and integrating in $\Omega_{C}$ and $\Omega_{I}$, respectively, we obtain by integration by parts

$$
\begin{aligned}
& \int_{\Omega_{C}}\left(\mu_{C}^{-1} \operatorname{rot} \mathbf{A}_{C} \cdot \operatorname{rot} \overline{\mathbf{v}_{C}}+\mu_{*}^{-1} \operatorname{div} \mathbf{A}_{C} \operatorname{div} \overline{\mathbf{v}_{C}}+i \omega \sigma \mathbf{A}_{C} \cdot \overline{\mathbf{v}_{C}}+i \omega \sigma \operatorname{grad} V_{C} \cdot \overline{\mathbf{v}_{C}}\right) \\
& \quad-\int_{\Gamma}\left[\left(\mu_{C}^{-1} \operatorname{rot} \mathbf{A}_{C}\right) \times \mathbf{n}_{C}\right] \cdot \overline{\mathbf{v}_{C}}-\mu_{*}^{-1} \int_{\Gamma} \overline{\mathbf{v}_{C}} \cdot \mathbf{n}_{C} \operatorname{div} \mathbf{A}_{C} \\
& \quad \int_{\Omega_{C}} \mathbf{J}_{e, C} \cdot \overline{\mathbf{v}_{C}} \\
& \int_{\Omega_{I}}\left(\mu_{I}^{-1} \operatorname{rot} \mathbf{A}_{I} \cdot \operatorname{rot} \overline{\mathbf{v}_{I}}+\mu_{*}^{-1} \operatorname{div} \mathbf{A}_{I} \operatorname{div} \overline{\mathbf{v}_{I}}\right) \\
& \quad-\int_{\Gamma}\left[\left(\mu_{I}^{-1} \operatorname{rot} \mathbf{A}_{I}\right) \times \mathbf{n}_{I}\right] \cdot \overline{\mathbf{v}_{I}}-\mu_{*}^{-1} \int_{\Gamma} \overline{\mathbf{v}_{I}} \cdot \mathbf{n}_{I} \operatorname{div} \mathbf{A}_{I} \\
& =\int_{\Omega_{I}} \mathbf{J}_{e, I} \cdot \overline{\mathbf{v}_{I}},
\end{aligned}
$$

having used $(2.5)_{7}$ and $(2.5)_{8}$.

Since $\mathbf{n}_{C} \times\left(\mathbf{v}_{C} \times \mathbf{n}_{C}\right)=\mathbf{n}_{I} \times\left(\mathbf{v}_{I} \times \mathbf{n}_{I}\right)$ on $\Gamma$ and $\mathbf{v}_{C} \cdot \mathbf{n}_{C}+\mathbf{v}_{I} \cdot \mathbf{n}_{I}=0$ on $\Gamma$, by adding these equations and taking into account the interface conditions $(2.5)_{9}$ and $(2.5)_{12}$ one has

$$
\begin{aligned}
\int_{\Omega_{C}}\left(\mu_{C}^{-1}\right. & \left.\operatorname{rot} \mathbf{A}_{C} \cdot \operatorname{rot} \overline{\mathbf{v}_{C}}+\mu_{*}^{-1} \operatorname{div} \mathbf{A}_{C} \operatorname{div} \overline{\mathbf{v}_{C}}\right) \\
& +\int_{\Omega_{C}}\left(i \omega \sigma \mathbf{A}_{C} \cdot \overline{\mathbf{v}_{C}}+i \omega \sigma \operatorname{grad} V_{C} \cdot \overline{\mathbf{v}_{C}}\right) \\
& +\int_{\Omega_{I}}\left(\mu_{I}^{-1} \operatorname{rot} \mathbf{A}_{I} \cdot \operatorname{rot} \overline{\mathbf{v}_{I}}+\mu_{*}^{-1} \operatorname{div} \mathbf{A}_{I} \operatorname{div} \overline{\mathbf{v}_{I}}\right) \\
= & \int_{\Omega_{C}} \mathbf{J}_{e, C} \cdot \overline{\mathbf{v}_{C}}+\int_{\Omega_{I}} \mathbf{J}_{e, I} \cdot \overline{\mathbf{v}_{I}} .
\end{aligned}
$$


Let us now multiply $(2.5)_{3}$ by $\overline{\phi_{C}}$ and integrate in $\Omega_{C}$ : by integration by parts we find

$$
\begin{array}{r}
\int_{\Omega_{C}}\left(i \omega \sigma \mathbf{A}_{C} \cdot \operatorname{grad} \overline{\phi_{C}}+i \omega \sigma \operatorname{grad} V_{C} \cdot \operatorname{grad} \overline{\phi_{C}}\right) \\
=\int_{\Omega_{C}} \mathbf{J}_{e, C} \cdot \operatorname{grad} \overline{\phi_{C}}+\int_{\Gamma} \mathbf{J}_{e, I} \cdot \mathbf{n}_{I} \overline{\phi_{C}}
\end{array}
$$

having used $(2.5)_{4}$.

Introducing the energy functional

$$
\begin{aligned}
\mathcal{A}\left[\left(\mathbf{A}, V_{C}\right)\right. & \left.,\left(\mathbf{v}, \phi_{C}\right)\right] \\
: & =\int_{\Omega}\left(\mu^{-1} \operatorname{rot} \mathbf{A} \cdot \operatorname{rot} \overline{\mathbf{v}}+\mu_{*}^{-1} \operatorname{div} \mathbf{A} \operatorname{div} \overline{\mathbf{v}}\right) \\
& +\int_{\Omega_{C}}\left(i \omega \sigma \mathbf{A}_{C} \cdot \overline{\mathbf{v}_{C}}+i \omega \sigma \operatorname{grad} V_{C} \cdot \overline{\mathbf{v}_{C}}\right) \\
& +\int_{\Omega_{C}}\left(i \omega \sigma \mathbf{A}_{C} \cdot \operatorname{grad} \overline{\phi_{C}}+i \omega \sigma \operatorname{grad} V_{C} \cdot \operatorname{grad} \overline{\phi_{C}}\right)
\end{aligned}
$$

we have finally rewritten $(2.5)$ as

$$
\begin{aligned}
& \text { find }\left(\mathbf{A}, V_{C}\right) \in W \times H_{\sharp}^{1}\left(\Omega_{C}\right): \\
& \qquad \begin{array}{l}
\mathcal{A}\left[\left(\mathbf{A}, V_{C}\right),\left(\mathbf{v}, \phi_{C}\right)\right] \\
=\int_{\Omega} \mathbf{J}_{e} \cdot \overline{\mathbf{v}}+\int_{\Omega_{C}} \mathbf{J}_{e, C} \cdot \operatorname{grad} \overline{\phi_{C}}+\int_{\Gamma} \mathbf{J}_{e, I} \cdot \mathbf{n}_{I} \overline{\phi_{C}} \\
\forall\left(\mathbf{v}, \phi_{C}\right) \in W \times H_{\sharp}^{1}\left(\Omega_{C}\right),
\end{array}
\end{aligned}
$$

where

$$
\begin{aligned}
W:=\{\mathbf{v} \in H(\operatorname{rot} ; \Omega) \cap H(\operatorname{div} ; \Omega) \mid \mathbf{v} \cdot \mathbf{n}=0 \text { on } \partial \Omega, \\
\left.\mathcal{G}_{k}(\mathbf{v})=0 \forall k=1, \ldots, n_{\partial \Omega}\right\},
\end{aligned}
$$

and

$$
H_{\sharp}^{1}\left(\Omega_{C}\right):=\left\{\phi_{C} \in H^{1}\left(\Omega_{C}\right) \mid \int_{\Omega_{C, j}} \phi_{C \mid \Omega_{C, j}}=0 \forall j=1, \ldots, p_{\Gamma}\right\} .
$$

\section{From the weak to the strong formulation}

Before starting the proof that the weak problem (3.4) has a unique solution (for that, see Section 5), it is useful to show that a solution to (3.4) is indeed a solution to $(2.5)$.

In this respect, note that the additional constraints $\mathcal{G}_{k}(\mathbf{A})=0$ for all $k=1, \ldots, n_{\partial \Omega}$ and $\int_{\Omega_{C, j}} \phi_{C \mid \Omega_{C, j}}=0$ for all $j=1, \ldots, p_{\Gamma}$ have not been used in deriving (3.4). Therefore, for going back from (3.4) to (2.5) it is sufficient to show that a solution to (3.4) satisfies the equation

$$
\mathcal{A}\left[\left(\mathbf{A}, V_{C}\right),\left(\mathbf{v}, \phi_{C}\right)\right]=\int_{\Omega} \mathbf{J}_{e} \cdot \overline{\mathbf{v}}+\int_{\Omega_{C}} \mathbf{J}_{e, C} \cdot \operatorname{grad} \overline{\phi_{C}}+\int_{\Gamma} \mathbf{J}_{e, I} \cdot \mathbf{n}_{I} \overline{\phi_{C}}
$$

also for any test function $\left(\mathbf{v}, \phi_{C}\right)$ such that $\mathbf{v} \in H(\operatorname{rot} ; \Omega) \cap H(\operatorname{div} ; \Omega)$ with $\mathbf{v} \cdot \mathbf{n}=0$ on $\partial \Omega$ and $\phi_{C} \in H^{1}\left(\Omega_{C}\right)$, namely, without any additional constraint. 
Thus, let $\left(\mathbf{A}, V_{C}\right)$ be a solution to (3.4). For any $\phi_{C} \in H^{1}\left(\Omega_{C}\right)$ set

$$
\phi_{j}^{*}:=\frac{1}{\operatorname{meas}\left(\Omega_{C, j}\right)} \int_{\Omega_{C, j}} \phi_{C \mid \Omega_{C, j}} ;
$$

clearly, the function $\phi_{C}-\phi_{C}^{*}$, where $\phi_{C}^{*}$ is defined as

$$
\phi_{C \mid \Omega_{C, j}}^{*}:=\phi_{j}^{*}
$$

belongs to $H_{\sharp}^{1}\left(\Omega_{C}\right)$. Therefore, for any $\left(\mathbf{v}, \phi_{C}\right) \in W \times H^{1}\left(\Omega_{C}\right)$ we have

$$
\begin{aligned}
\mathcal{A}\left[\left(\mathbf{A}, V_{C}\right),\left(\mathbf{v}, \phi_{C}\right)\right]= & \mathcal{A}\left[\left(\mathbf{A}, V_{C}\right),\left(\mathbf{v}, \phi_{C}-\phi_{C}^{*}\right)\right] \\
= & \int_{\Omega} \mathbf{J}_{e} \cdot \overline{\mathbf{v}}+\int_{\Omega_{C}} \mathbf{J}_{e, C} \cdot \operatorname{grad} \overline{\phi_{C}}+\int_{\Gamma} \mathbf{J}_{e, I} \cdot \mathbf{n}_{I} \overline{\phi_{C}} \\
& \quad-\sum_{j=1}^{p_{\Gamma}} \overline{\phi_{j}^{*}} \int_{\Gamma_{j}} \mathbf{J}_{e, I} \cdot \mathbf{n}_{I} \\
= & \int_{\Omega} \mathbf{J}_{e} \cdot \overline{\mathbf{v}}+\int_{\Omega_{C}} \mathbf{J}_{e, C} \cdot \operatorname{grad} \overline{\phi_{C}}+\int_{\Gamma} \mathbf{J}_{e, I} \cdot \mathbf{n}_{I} \overline{\phi_{C}},
\end{aligned}
$$

as by assumption we have $\int_{\Gamma_{j}} \mathbf{J}_{e, I} \cdot \mathbf{n}_{I}=0$ for each $j=1, \ldots, p_{\Gamma}-1$, and moreover $\operatorname{div} \mathbf{J}_{e, I}=0$ in $\Omega_{I}$ and $\mathbf{J}_{e, I} \cdot \mathbf{n}=0$ on $\partial \Omega$, so that $\int_{\Gamma_{j}} \mathbf{J}_{e, I} \cdot \mathbf{n}_{I}=0$ for $j=p_{\Gamma}$, too.

Taking $\mathbf{v}=\mathbf{0}$, as a first result we have therefore that any solution to (3.4) satisfies

$$
\begin{array}{ll}
\operatorname{div}\left(i \omega \sigma \mathbf{A}_{C}+i \omega \sigma \operatorname{grad} V_{C}\right)=\operatorname{div} \mathbf{J}_{e, C} & \text { in } \Omega_{C} \\
\left(i \omega \sigma \mathbf{A}_{C}+i \omega \sigma \operatorname{grad} V_{C}\right) \cdot \mathbf{n}_{C}=\mathbf{J}_{e, C} \cdot \mathbf{n}_{C}+\mathbf{J}_{e, I} \cdot \mathbf{n}_{I} & \text { on } \Gamma
\end{array}
$$

Setting

$$
\mathbf{J}:=\left\{\begin{array}{ll}
-i \omega \sigma \mathbf{A}_{C}-i \omega \sigma \operatorname{grad} V_{C}+\mathbf{J}_{e, C} & \text { in } \Omega_{C} \\
\mathbf{J}_{e, I} & \text { in } \Omega_{I}
\end{array},\right.
$$

the assumptions $\operatorname{div} \mathbf{J}_{e, I}=0$ in $\Omega_{I}$ and $\mathbf{J}_{e, I} \cdot \mathbf{n}=0$ on $\partial \Omega$ are telling us that $\operatorname{div} \mathbf{J}=0$ in $\Omega$ and $\mathbf{J} \cdot \mathbf{n}=0$ on $\partial \Omega$.

For any $\mathbf{v} \in H(\operatorname{rot} ; \Omega) \cap H(\operatorname{div} ; \Omega)$ with $\mathbf{v} \cdot \mathbf{n}=0$ on $\partial \Omega$, let us define now by $\mathbf{v}_{m}$ the harmonic field in $\mathcal{H}(m ; \Omega)$ (see (2.2)) satisfying $\mathcal{G}_{z}\left(\mathbf{v}_{m}\right)=\mathcal{G}_{z}(\mathbf{v})$ for each $z=1, \ldots, n_{\partial \Omega}^{*}$.

The vector $\mathbf{v}_{m}$ is well-defined provided that the following condition holds:

$$
\begin{aligned}
& \text { for } \mathbf{w}_{m} \in \mathcal{H}(m ; \Omega), \text { the conditions } \mathcal{G}_{z}\left(\mathbf{w}_{m}\right)=0 \\
& \text { for each } z=1, \ldots, n_{\partial \Omega}^{*} \text { give } \mathbf{w}_{m}=\mathbf{0} \text { in } \Omega .
\end{aligned}
$$

We will see later on how to choose the functional $\mathcal{G}_{z}(\cdot)$ in such a way that this condition is satisfied.

Clearly, the difference $\mathbf{v}-\mathbf{v}_{m}$ belongs to $W$. Hence

$$
\begin{aligned}
\mathcal{A}\left[\left(\mathbf{A}, V_{C}\right),\left(\mathbf{v}, \phi_{C}\right)\right] & \\
= & \mathcal{A}\left[\left(\mathbf{A}, V_{C}\right),\left(\mathbf{v}-\mathbf{v}_{m}, \phi_{C}\right)\right]+\mathcal{A}\left[\left(\mathbf{A}, V_{C}\right),\left(\mathbf{v}_{m}, 0\right)\right] \\
= & \int_{\Omega} \mathbf{J}_{e} \cdot\left(\overline{\mathbf{v}}-\overline{\mathbf{v}_{m}}\right)+\int_{\Omega_{C}} \mathbf{J}_{e, C} \cdot \operatorname{grad} \overline{\phi_{C}}+\int_{\Gamma} \mathbf{J}_{e, I} \cdot \mathbf{n}_{I} \overline{\phi_{C}} \\
& \quad+\int_{\Omega_{C}}\left(i \omega \sigma \mathbf{A}_{C}+i \omega \sigma \operatorname{grad} V_{C}\right) \cdot \overline{\mathbf{v}_{m, C}} \\
= & \int_{\Omega} \mathbf{J}_{e} \cdot \overline{\mathbf{v}}+\int_{\Omega_{C}} \mathbf{J}_{e, C} \cdot \operatorname{grad} \overline{\phi_{C}}+\int_{\Gamma} \mathbf{J}_{e, I} \cdot \mathbf{n}_{I} \overline{\phi_{C}} \\
\quad & \quad-\int_{\Omega} \mathbf{J} \cdot \overline{\mathbf{v}_{m}} .
\end{aligned}
$$


Therefore, the only result that remains to be proved is

$$
\int_{\Omega} \mathbf{J} \cdot \overline{\mathbf{v}_{m}}=0 .
$$

Let us introduce now the real vector functions $\boldsymbol{\eta}_{z}$, the basis of the space $\mathcal{H}(m ; \Omega)$ such that $\mathcal{G}_{q}\left(\boldsymbol{\eta}_{z}\right)=\delta_{q z}$. Clearly, $\boldsymbol{\eta}_{z} \in W$ for $z=n_{\partial \Omega}+1, \ldots, n_{\partial \Omega}^{*}$. Using these test functions (and $\phi_{C}=0$ ) we find

$$
\int_{\Omega} \mathbf{J} \cdot \boldsymbol{\eta}_{z}=0 \quad \forall z=n_{\partial \Omega}+1, \ldots, n_{\partial \Omega}^{*} .
$$

Therefore, writing the vector field $\mathbf{v}_{m}$ in term of the basis $\boldsymbol{\eta}_{z}$ as $\mathbf{v}_{m}=\sum_{z} \alpha_{z} \boldsymbol{\eta}_{z}$, we have

$$
\int_{\Omega} \mathbf{J} \cdot \overline{\mathbf{v}_{m}}=\sum_{z=1}^{n_{\partial \Omega}^{*}} \overline{\alpha_{z}} \int_{\Omega} \mathbf{J} \cdot \boldsymbol{\eta}_{z}=\sum_{k=1}^{n_{\partial \Omega}} \overline{\alpha_{k}} \int_{\Omega} \mathbf{J} \cdot \boldsymbol{\eta}_{k} .
$$

The problem now is to show that

$$
\int_{\Omega} \mathbf{J} \cdot \boldsymbol{\eta}_{k}=0 \quad \forall k=1, \ldots, n_{\partial \Omega} .
$$

We have not yet used the assumptions

$$
\int_{\Omega_{I}} \mathbf{J}_{e, I} \cdot \boldsymbol{\pi}_{k, I}=0 \quad \forall k=1, \ldots, n_{\partial \Omega} .
$$

We know that any vector field $\boldsymbol{\pi}_{k, I}$ can be expressed as the $\left(L^{2}\left(\Omega_{I}\right)\right)^{3}$-extension of $\operatorname{grad} p_{k, I}$, where $p_{k, I}$, defined in $\Omega_{I} \backslash \Sigma_{k}$, is the real function solution of

$$
\begin{cases}\operatorname{div}\left(\varepsilon_{I} \operatorname{grad} p_{k, I}\right)=0 & \text { in } \Omega_{I} \backslash \Sigma_{k} \\ \varepsilon_{I} \operatorname{grad} p_{k, I} \cdot \mathbf{n}_{I}=0 & \text { on } \partial \Omega \backslash \partial \Sigma_{k} \\ p_{k, I}=0 & \text { on } \Gamma \\ {\left[\varepsilon_{I} \operatorname{grad} p_{k, I} \cdot \mathbf{n}_{\Sigma}\right]_{\Sigma_{k}}=0} & \\ {\left[p_{k, I}\right]_{\Sigma_{k}}=1,} & \end{cases}
$$

having denoted by $[\cdot]_{\Sigma_{k}}$ the jump across the surface $\Sigma_{k}$ (see, e.g., Foias and Temam [15]). Therefore, since $\operatorname{div} \mathbf{J}_{e, I}=0$ in $\Omega_{I}$ and $\mathbf{J}_{e, I} \cdot \mathbf{n}=0$ on $\partial \Omega$,

$$
\begin{aligned}
0=\int_{\Omega_{I}} \mathbf{J}_{e, I} \cdot \boldsymbol{\pi}_{k, I} & =\int_{\Omega_{I} \backslash \Sigma_{k}} \mathbf{J}_{e, I} \cdot \operatorname{grad} p_{k, I} \\
& =-\int_{\Omega_{I} \backslash \Sigma_{k}}\left(\operatorname{div} \mathbf{J}_{e, I}\right) p_{k, I}+\int_{\partial \Omega_{I} \backslash \partial \Sigma_{k}} \mathbf{J}_{e, I} \cdot \mathbf{n}_{I} p_{k, I} \\
& +\int_{\Sigma_{k}} \mathbf{J}_{e, I} \cdot \mathbf{n}_{\Sigma}\left[p_{k, I}\right]_{\Sigma_{k}} \\
& =\int_{\Sigma_{k}} \mathbf{J}_{e, I} \cdot \mathbf{n}_{\Sigma} .
\end{aligned}
$$


Let us remark now that another set of basis functions of $\mathcal{H}(m ; \Omega)$ is given by $\boldsymbol{\pi}_{z}^{*}$, the $\left(L^{2}(\Omega)\right)^{3}$-extension of $\operatorname{grad} p_{z}^{*}$, where $p_{z}^{*}$, defined in $\Omega \backslash \Sigma_{z}^{*}$, is the real function solution of

$$
\begin{cases}\Delta p_{z}^{*}=0 & \text { in } \Omega \backslash \Sigma_{z}^{*} \\ \operatorname{grad} p_{z}^{*} \cdot \mathbf{n}=0 & \text { on } \partial \Omega \backslash \partial \Sigma_{z}^{*} \\ {\left[\operatorname{grad} p_{z}^{*} \cdot \mathbf{n}_{\Sigma}\right]_{\Sigma_{z}^{*}}=0} & \\ {\left[p_{z}^{*}\right]_{\Sigma_{z}^{*}}=1,} & \end{cases}
$$

having denoted by $[\cdot]_{\Sigma_{z}^{*}}$ the jump across the surface $\Sigma_{z}^{*}$. Hence, proceeding as before, and recalling that $\operatorname{div} \mathbf{J}=0$ in $\Omega, \mathbf{J} \cdot \mathbf{n}=0$ on $\partial \Omega$ and that the "cuts" satisfy $\Sigma_{k} \subset \Omega_{I}$ for $k=1, \ldots, n_{\partial \Omega}$, we find that

$$
0=\int_{\Sigma_{k}} \mathbf{J}_{e, I} \cdot \mathbf{n}_{\Sigma}=\int_{\Sigma_{k}} \mathbf{J} \cdot \mathbf{n}_{\Sigma}=\int_{\Omega} \mathbf{J} \cdot \boldsymbol{\pi}_{k}^{*} \quad \forall k=1, \ldots, n_{\partial \Omega} .
$$

We can write the basis $\boldsymbol{\pi}_{z}^{*}$ in terms of the basis $\boldsymbol{\eta}_{z}$ :

$$
\boldsymbol{\pi}_{q}^{*}=\sum_{z=1}^{n_{\partial \Omega}^{*}} \beta_{q z} \boldsymbol{\eta}_{z}
$$

Hence, using (4.5), for $k=1, \ldots, n_{\partial \Omega}$ one has

$$
0=\int \mathbf{J} \cdot \boldsymbol{\pi}_{k}^{*}=\sum_{z=1}^{n_{\partial \Omega}^{*}} \beta_{k z} \int_{\Omega} \mathbf{J} \cdot \boldsymbol{\eta}_{z}=\sum_{i=1}^{n_{\partial \Omega}} \beta_{k i} \int_{\Omega} \mathbf{J} \cdot \boldsymbol{\eta}_{i}
$$

Let us assume now that the set of linear constraints $\mathcal{G}_{z}(\cdot)$ is such that the following condition is satisfied:

the basis $\boldsymbol{\pi}_{q}^{*}$ can be expressed in terms of the basis $\boldsymbol{\eta}_{\boldsymbol{z}}$ associated to the linear functionals $\mathcal{G}_{z}(\cdot)$ by means of a matrix $\left\{\beta_{q z}\right\}, q, z=1, \ldots, n_{\partial \Omega}^{*}$, such that the $n_{\partial \Omega} \times n_{\partial \Omega}$ sub-matrix $\left\{\beta_{k i}\right\}$ is non-singular.

As a consequence, from (4.8) it follows that (4.6) holds.

Therefore, we can finally conclude that, if the functionals $\mathcal{G}_{z}(\cdot)$ verify $(C 1)$ and $(C 2)$, then any solution to (3.4) indeed satisfies equation (4.1) for any test function $\left(\mathbf{v}, \phi_{C}\right)$ such that $\mathbf{v} \in H(\operatorname{rot} ; \Omega) \cap H(\operatorname{div} ; \Omega)$ with $\mathbf{v} \cdot \mathbf{n}=0$ on $\partial \Omega$ and $\phi_{C} \in H^{1}\left(\Omega_{C}\right)$, and therefore any solution of the weak problem (3.4) is a solution of the strong problem (2.5), hence of the original problem (2.4).

Let us finish this Section by showing some examples of choices of the linear functionals $\mathcal{G}_{z}(\cdot)$ for which conditions $(C 1)$ and $(C 2)$ are satisfied.

Case 1: $n_{\partial \Omega}=0$. 
This is the simplest case: no constraints have to be imposed in (2.5) (or, equivalently, in the space $W$ ).

A geometric example of this situation is the case in which $\Omega$ and $\Omega_{C}$ are two ball-like sets $\left(n_{\partial \Omega}=0, n_{\partial \Omega}^{*}=0\right)$, or two co-axial tori $\left(n_{\partial \Omega}=0, n_{\partial \Omega}^{*}=1\right)$.

Case 2: $0<n_{\partial \Omega}=n_{\partial \Omega}^{*}$.

Also this case is simple: in fact, condition $(C 2)$ is clearly satisfied, as the matrix $\left\{\beta_{k i}\right\}$ is now expressing the change of basis, therefore is non-singular for any choice of $\mathcal{G}_{k}(\cdot)$ satisfying $(C 1)$. Concerning this condition, it is satisfied with anyone of the following choices:

$$
\mathcal{G}_{k}(\mathbf{v})=\int_{\Sigma_{k}} \mathbf{v} \cdot \mathbf{n}_{\Sigma} ; \mathcal{G}_{k}(\mathbf{v})=\int_{\Omega} \mathbf{v} \cdot \boldsymbol{\pi}_{k}^{*} ; \mathcal{G}_{k}(\mathbf{v})=\int_{\Omega} \mathbf{v} \cdot \boldsymbol{\Psi}_{k}^{*} .
$$

Here $\boldsymbol{\Psi}_{k}^{*}$ is the $\left(L^{2}(\Omega)\right)^{3}$-extension of $\operatorname{grad} \psi_{k}^{*}$, where $\psi_{k}^{*}$ is any (continuous and piecewise regular) real function, multivalued on $\Sigma_{k}^{*}$, that satisfies $\left[\psi_{k}^{*}\right]_{\Sigma_{k}^{*}}=1$.

Using the fact that both $\boldsymbol{\pi}_{k}^{*}$ and $\boldsymbol{\Psi}_{k}^{*}$ are the extensions of the gradient of a function having jump equal to 1 on $\Sigma_{k}^{*}$, it is easily seen that, for a divergence free and tangential vector field, the three constraints above are the same, and all of them express the orthogonality to $\mathcal{H}(m ; \Omega)$. Therefore, the solution $\mathbf{A}$ to $(3.4)$ will be the same for any choice of $\mathcal{G}_{k}(\cdot)$; the only difference is, when approximating the solution by means of finite elements, the algebraic structure of the stiffness matrix associated to (3.4).

A geometric example of this situation is the case in which $\Omega$ is a torus and $\Omega_{C}$ is a ball-like set $\left(n_{\partial \Omega}=1, n_{\partial \Omega}^{*}=1\right)$.

Case 3: $0<n_{\partial \Omega}<n_{\partial \Omega}^{*}$.

This case seems to be more difficult to treat. In fact, condition $(C 2)$ is not easy to be verified. We propose three alternative choices of the linear functionals $\mathcal{G}_{z}(\cdot)$ for which the associated basis functions $\boldsymbol{\eta}_{z}$ are exactly the basis functions $\boldsymbol{\pi}_{z}^{*}$, so that $(C 2)$ is clearly satisfied. To be clear, let us introduce the matrix $\left\{\gamma_{q z}\right\}$ given by $\gamma_{q z}:=\int_{\Omega} \boldsymbol{\pi}_{q}^{*} \cdot \boldsymbol{\pi}_{z}^{*}$, and denote by $\left\{\theta_{q z}\right\}$ its inverse matrix. For $z=1, \ldots, n_{\partial \Omega}^{*}$, we propose one of the three functionals:

$$
\begin{aligned}
& \mathcal{G}_{z}(\mathbf{v}):=\sum_{p=1}^{n_{\partial \Omega}^{*}} \theta_{z p} \int_{\Sigma_{p}^{*}} \mathbf{v} \cdot \mathbf{n}_{\Sigma} ; \mathcal{G}_{z}(\mathbf{v}):=\sum_{p=1}^{n_{\partial \Omega}^{*}} \theta_{z p} \int_{\Omega} \mathbf{v} \cdot \boldsymbol{\pi}_{p}^{*} \\
& \mathcal{G}_{z}(\mathbf{v}):=\sum_{p=1}^{n_{\partial \Omega}^{*}} \theta_{z p} \int_{\Omega} \mathbf{v} \cdot \boldsymbol{\Psi}_{p}^{*} .
\end{aligned}
$$

As we have already noted, the functionals above are the same for a divergence free and tangential vector field. Therefore, we check that the associated basis functions are $\boldsymbol{\pi}_{z}^{*}$ only for the second one; indeed, we find

$$
\mathcal{G}_{z}\left(\boldsymbol{\pi}_{q}^{*}\right)=\sum_{p=1}^{n_{\partial \Omega}^{*}} \theta_{z p} \int_{\Omega} \boldsymbol{\pi}_{q}^{*} \cdot \boldsymbol{\pi}_{p}^{*}=\sum_{p=1}^{n_{\partial \Omega}^{*}} \theta_{z p} \gamma_{p q}=\delta_{q z} .
$$

Moreover, if $\mathbf{w}_{m} \in \mathcal{H}(m ; \Omega)$, we can write $\mathbf{w}_{m}=\sum_{q} \alpha_{q} \boldsymbol{\pi}_{q}^{*}$, therefore

$$
\mathcal{G}_{z}\left(\mathbf{w}_{m}\right)=\sum_{q=1}^{n_{\partial \Omega}^{*}} \alpha_{q} \mathcal{G}_{z}\left(\boldsymbol{\pi}_{q}^{*}\right)=\alpha_{z}
$$


hence condition $(C 1)$ is satisfied.

A geometric example of this situation is the case in which $\Omega$ is a double torus and $\Omega_{C}$ is a torus, co-axial to one of the two handles of $\Omega\left(n_{\partial \Omega}=1\right.$, $\left.n_{\partial \Omega}^{*}=2\right)$.

\section{Existence and uniqueness of the solution to the weak formulation}

The proof of existence and uniqueness is different in the three cases devised at the end of Section 4. Let us recall that here and in the sequel we assume that the functionals $\mathcal{G}_{z}(\cdot)$ are as in (4.9) or (4.10).

Case 2 (or Case 1 with $0=n_{\partial \Omega}=n_{\partial \Omega}^{*}$ ).

In these cases, the existence and uniqueness result derives from the LaxMilgram lemma (see, e.g., Bossavit [7], p. 313; Dautray and Lions [12], Volume 2, Chapter VI, Section 3, Theorem 7 and Remark 8). Since $W \times H_{\sharp}^{1}\left(\Omega_{C}\right)$ is clearly a Hilbert space with respect to the natural scalar product, and the sesquilinear form $\mathcal{A}[\cdot, \cdot]$ is continuous in $\left(W \times H_{\sharp}^{1}\left(\Omega_{C}\right)\right) \times\left(W \times H_{\sharp}^{1}\left(\Omega_{C}\right)\right)$, we have only to check that the right hand side in (3.4) is a continuous (antilinear) functional in $W \times H_{\sharp}^{1}\left(\Omega_{C}\right)$ and that $\mathcal{A}[\cdot, \cdot]$ is coercive in $\left(W \times H_{\sharp}^{1}\left(\Omega_{C}\right)\right) \times\left(W \times H_{\sharp}^{1}\left(\Omega_{C}\right)\right)$, namely, that there exists a constant $\kappa_{0}>0$ such that for each $\left(\mathbf{v}, \phi_{C}\right) \in W \times$ $H_{\sharp}^{1}\left(\Omega_{C}\right)$

$$
\begin{aligned}
\left|\mathcal{A}\left[\left(\mathbf{v}, \phi_{C}\right),\left(\mathbf{v}, \phi_{C}\right)\right]\right| \geq \kappa_{0}\left(\int _ { \Omega } \left(|\mathbf{v}|^{2}+\mid\right.\right. & \left.\left.\operatorname{rot} \mathbf{v}\right|^{2}+|\operatorname{div} \mathbf{v}|^{2}\right) \\
& \left.+\int_{\Omega_{C}}\left(\left|\phi_{C}\right|^{2}+\left|\operatorname{grad} \phi_{C}\right|^{2}\right)\right) .
\end{aligned}
$$

In the right hand side of (3.4) the only term to check is the third one. We have

$$
\begin{aligned}
\left|\int_{\Gamma} \mathbf{J}_{e, I} \cdot \mathbf{n}_{I} \overline{\phi_{C}}\right| & \leq C_{1}\left\|\mathbf{J}_{e, I} \cdot \mathbf{n}_{I}\right\|_{H^{-1 / 2}(\Gamma)}\left\|\phi_{C}\right\|_{H^{1 / 2}(\Gamma)} \\
& \leq C_{2}\left\|\mathbf{J}_{e, I}\right\|_{L^{2}\left(\Omega_{I}\right)}\left\|\phi_{C}\right\|_{H^{1}\left(\Omega_{C}\right)},
\end{aligned}
$$

having used the trace theorems from $H\left(\operatorname{div} ; \Omega_{I}\right)$ onto $H^{-1 / 2}(\Gamma)$ and from $H^{1}\left(\Omega_{C}\right)$ onto $H^{1 / 2}(\Gamma)$ (see, for instance, Girault and Raviart [16], Chapter I, Theorems 2.5 and 1.5 , respectively).

Concerning (5.1), we have:

$$
\begin{aligned}
\mathcal{A}\left[\left(\mathbf{v}, \phi_{C}\right),\left(\mathbf{v}, \phi_{C}\right)\right]=\int_{\Omega}( & \left.\mu^{-1} \operatorname{rot} \mathbf{v} \cdot \operatorname{rot} \overline{\mathbf{v}}+\mu_{*}^{-1}|\operatorname{div} \mathbf{v}|^{2}\right) \\
& +i \omega \int_{\Omega_{C}}\left(\sigma \mathbf{v}_{C} \cdot \overline{\mathbf{v}_{C}}+\sigma \operatorname{grad} \phi_{C} \cdot \operatorname{grad} \overline{\phi_{C}}\right. \\
& \left.+\sigma \operatorname{grad} \phi_{C} \cdot \overline{\mathbf{v}_{C}}+\sigma \mathbf{v}_{C} \cdot \operatorname{grad} \overline{\phi_{C}}\right) .
\end{aligned}
$$

Since

$$
\sigma \operatorname{grad} \phi_{C} \cdot \overline{\mathbf{v}_{C}}+\sigma \mathbf{v}_{C} \cdot \operatorname{grad} \overline{\phi_{C}}=2 \operatorname{Re}\left(\sigma \operatorname{grad} \phi_{C} \cdot \overline{\mathbf{v}_{C}}\right),
$$


we have

$$
\begin{aligned}
\left|\mathcal{A}\left[\left(\mathbf{v}, \phi_{C}\right),\left(\mathbf{v}, \phi_{C}\right)\right]\right|^{2} & \\
=\left(\int _ { \Omega } \left(\mu^{-1} \operatorname{rot} \mathbf{v} \cdot \operatorname{rot} \overline{\mathbf{v}}+\right.\right. & \left.\left.\mu_{*}^{-1}|\operatorname{div} \mathbf{v}|^{2}\right)\right)^{2} \\
+\omega^{2}\left(\int _ { \Omega _ { C } } \left[\sigma \mathbf{v}_{C} \cdot \overline{\mathbf{v}_{C}}\right.\right. & +\sigma \operatorname{grad} \phi_{C} \cdot \operatorname{grad} \overline{\phi_{C}} \\
& \left.\left.+2 \operatorname{Re}\left(\sigma \operatorname{grad} \phi_{C} \cdot \overline{\mathbf{v}_{C}}\right)\right]\right)^{2}
\end{aligned}
$$

On the other hand, given a couple of real numbers $a$ and $b$ we have

$$
a^{2}=(a+b-b)^{2} \leq 2(a+b)^{2}+2 b^{2}
$$

therefore

$$
(a+b)^{2} \geq \frac{1}{2} a^{2}-b^{2}
$$

and also

$$
(a+b)^{2} \geq 2 \rho(a+b)^{2} \geq \rho a^{2}-2 \rho b^{2}
$$

for each $0<\rho \leq 1 / 2$.

Hence

$$
\begin{gathered}
\omega^{2}\left(\int_{\Omega_{C}}\left[\sigma \mathbf{v}_{C} \cdot \overline{\mathbf{v}_{C}}+\sigma \operatorname{grad} \phi_{C} \cdot \operatorname{grad} \overline{\phi_{C}}+2 \operatorname{Re}\left(\sigma \operatorname{grad} \phi_{C} \cdot \overline{\mathbf{v}_{C}}\right)\right]\right)^{2} \\
\geq \omega^{2} \rho\left(\int_{\Omega_{C}}\left(\sigma \mathbf{v}_{C} \cdot \overline{\mathbf{v}_{C}}+\sigma \operatorname{grad} \phi_{C} \cdot \operatorname{grad} \overline{\phi_{C}}\right)\right)^{2} \\
\quad-8 \omega^{2} \rho\left|\int_{\Omega_{C}} \sigma \operatorname{grad} \phi_{C} \cdot \overline{\mathbf{v}_{C}}\right|^{2} \\
\geq \omega^{2} \rho\left(\int_{\Omega_{C}} \sigma \mathbf{v}_{C} \cdot \overline{\mathbf{v}_{C}}\right)^{2}+\omega^{2} \rho\left(\int_{\Omega_{C}} \sigma \operatorname{grad} \phi_{C} \cdot \operatorname{grad} \overline{\phi_{C}}\right)^{2} \\
\quad-\frac{\omega^{2} \rho}{2}\left(\int_{\Omega_{C}} \sigma \operatorname{grad} \phi_{C} \cdot \operatorname{grad} \overline{\phi_{C}}\right)^{2}-32 \omega^{2} \rho\left(\int_{\Omega_{C}} \sigma \mathbf{v}_{C} \cdot \overline{\mathbf{v}_{C}}\right)^{2} \\
\geq \frac{\omega^{2} \rho}{2} \sigma_{\min }^{2}\left(\int_{\Omega_{C}}\left|\operatorname{grad} \phi_{C}\right|^{2}\right)^{2}-31 \omega^{2} \rho \sigma_{\max }^{2}\left(\int_{\Omega_{C}}\left|\mathbf{v}_{C}\right|^{2}\right)^{2},
\end{gathered}
$$

where $\sigma_{\max }$ and $\sigma_{\min }$ are the maximum and minimum eigenvalue of $\sigma(\mathbf{x})$ in $\Omega_{C}$, respectively, and in the second step of the proof we have used the CauchySchwarz inequality and the estimate $|a b| \leq \frac{1}{16} a^{2}+4 b^{2}$.

Using the Poincaré inequality (see, for instance, Dautray and Lions [12], Volume 2, Chapter IV, Section 7, Proposition 2), one can also conclude that there exists a constant $K_{1}>0$ such that

$$
\begin{aligned}
\int_{\Omega_{C}}\left|\operatorname{grad} \phi_{C}\right|^{2} & =\sum_{j=1}^{p_{\Gamma}} \int_{\Omega_{C, j}}\left|\operatorname{grad} \phi_{C \mid \Omega_{C, j}}\right|^{2} \\
& \geq K_{1} \sum_{j=1}^{p_{\Gamma}} \int_{\Omega_{C, j}}\left(\left|\operatorname{grad} \phi_{C \mid \Omega_{C, j}}\right|^{2}+\left|\phi_{C \mid \Omega_{C, j}}\right|^{2}\right) \\
& =K_{1} \int_{\Omega_{C}}\left(\left|\operatorname{grad} \phi_{C}\right|^{2}+\left|\phi_{C}\right|^{2}\right) .
\end{aligned}
$$

Moreover, and this is the point in which Case 2 (or Case 1 with $0=n_{\partial \Omega}=$ $\left.n_{\partial \Omega}^{*}\right)$ differs from the other cases, there exists a constant $K_{2}>0$ such that for 
any function $\mathbf{v} \in W$ one has

$$
\begin{aligned}
& \left(\int_{\Omega}\left(\mu^{-1} \operatorname{rot} \mathbf{v} \cdot \operatorname{rot} \overline{\mathbf{v}}+\mu_{*}^{-1}|\operatorname{div} \mathbf{v}|^{2}\right)\right)^{2} \\
& \quad \geq\left(\int_{\Omega}\left(\mu_{\max }^{-1}|\operatorname{rot} \mathbf{v}|^{2}+\mu_{*}^{-1}|\operatorname{div} \mathbf{v}|^{2}\right)\right)^{2} \\
& \quad \geq K_{2}^{2}\left(\int_{\Omega}\left(|\operatorname{rot} \mathbf{v}|^{2}+|\operatorname{div} \mathbf{v}|^{2}+|\mathbf{v}|^{2}\right)\right)^{2},
\end{aligned}
$$

where $\mu_{\max }$ is the maximum eigenvalue of $\mu(\mathbf{x})$ in $\Omega$ (see, for instance, Girault and Raviart [16], Chapter I, Lemma 3.6; the proof can be easily extended to the present geometric situation, by proceeding as in Alonso and Valli [1], Lemma 3.3 , and noting that, for a divergence free and tangential vector field and for $\mathcal{G}_{k}(\cdot)$ defined as in $(4.9)$, the conditions $\mathcal{G}_{k}(\mathbf{v})=0$ for $k=1, \ldots, n_{\partial \Omega}=n_{\partial \Omega}^{*}$ are equivalent to the orthogonality to $\mathcal{H}(m ; \Omega)$ ).

Choosing $\rho$ such that $31 \omega^{2} \sigma_{\max }^{2} \rho<K_{2}^{2}$ and $0<\rho \leq 1 / 2$, from (5.3)-(5.6) we find at once (5.1).

(Estimate (5.1) has been claimed by Bossavit [8], [9], who considered the Coulomb gauged and the Lorenz gauged vector potential formulations of the eddy-current problem, respectively; however, his proof does not seem to be complete, and it is valid only for small value of $\omega$. Indeed, for completing the proof it seems necessary to take into account the crucial estimate (5.6).)

Case 3 (or Case 1 with $0=n_{\partial \Omega}<n_{\partial \Omega}^{*}$ ).

In this case coerciveness of $\mathcal{A}[\cdot, \cdot]$ in $\left(W \times H_{\sharp}^{1}\left(\Omega_{C}\right)\right) \times\left(W \times H_{\sharp}^{1}\left(\Omega_{C}\right)\right)$ is questionable, though we find that $\mathcal{A}\left[\left(\mathbf{v}, \phi_{C}\right),\left(\mathbf{v}, \phi_{C}\right)\right]=0$ implies $\mathbf{v}=\mathbf{0}$ and $\phi_{C}=0$, or, in other words, that $\left|\mathcal{A}\left[\left(\mathbf{v}, \phi_{C}\right),\left(\mathbf{v}, \phi_{C}\right)\right]\right|>0$ for $\left(\mathbf{v}, \phi_{C}\right) \neq(\mathbf{0}, 0)$.

This can be done as follows: assuming that $\mathcal{A}\left[\left(\mathbf{v}, \phi_{C}\right),\left(\mathbf{v}, \phi_{C}\right)\right]=0$, from (5.3) we have that $\operatorname{rot} \mathbf{v}=\mathbf{0}$ and $\operatorname{div} \mathbf{v}=0$ in $\Omega$, therefore $\mathbf{v} \in \mathcal{H}(m ; \Omega)$. Writing $\mathbf{v}$ in term of the basis functions of $\mathcal{H}(m ; \Omega)$, taking into account the definition of $\mathcal{G}_{k}(\cdot)$ in $(4.10)$ and that $\mathcal{G}_{k}(\mathbf{v})=0$ for $k=1, \ldots, n_{\partial \Omega}$, we also have

$$
\begin{aligned}
0 & =\mathcal{G}_{k}(\mathbf{v})=\mathcal{G}_{k}\left(\sum_{z=1}^{n_{\partial \Omega}^{*}} \alpha_{z} \boldsymbol{\pi}_{z}^{*}\right)=\sum_{p=1}^{n_{\partial \Omega}^{*}} \theta_{k p} \int_{\Omega} \sum_{z=1}^{n_{\partial \Omega}^{*}} \alpha_{z} \boldsymbol{\pi}_{z}^{*} \cdot \boldsymbol{\pi}_{p}^{*} \\
& =\sum_{z, p=1}^{n_{\partial \Omega}^{*}} \alpha_{z} \theta_{k p} \gamma_{p z}=\alpha_{k}, k=1, \ldots, n_{\partial \Omega} .
\end{aligned}
$$

From (5.3) we also find $\mathbf{v}_{C}+\operatorname{grad} \phi_{C}=\mathbf{0}$ in $\Omega_{C}$, namely,

$$
\sum_{z=n_{\partial \Omega}+1}^{n_{\partial \Omega}^{*}} \alpha_{z} \pi_{z, C}^{*}+\operatorname{grad} \phi_{C}=\mathbf{0} .
$$

Taking the line integral of $\mathbf{v}_{C}+\operatorname{grad} \phi_{C}=\mathbf{0}$ along the singular cycle contained in $\Omega_{C}$ and associated to the cutting surface $\Sigma_{q}^{*}, q=n_{\partial \Omega}+1, \ldots, n_{\partial \Omega}^{*}$, we find that the coefficient $\alpha_{q}$ is vanishing, therefore $\mathbf{v}=\mathbf{0}$ in $\Omega$ and consequently $\phi_{C}=0$ in $\Omega_{C}$. 
This is the proof of the uniqueness of the solution. Very likely, the proof of the existence can follow by the Fredholm alternative theory, or by passing to a saddle point formulation for expressing the constraints $\mathcal{G}_{k}(\mathbf{A})=0$; however, we will not dwell on it in the sequel.

Remark 5.1. It should be noted that, even if the constraints $\mathcal{G}_{k}(\mathbf{A})=0$ for $k=1, \ldots, n_{\partial \Omega}$ are not imposed, from $\mathcal{A}\left[\left(\mathbf{A}, V_{C}\right),\left(\mathbf{A}, V_{C}\right)\right]=0$ and $(5.3)$ we always obtain $\mathbf{A} \in \mathcal{H}(m ; \Omega)$ and $\mathbf{A}_{C}+\operatorname{grad} V_{C}=\mathbf{0}$ in $\Omega_{C}$. Therefore, $\mathbf{B}=\operatorname{rot} \mathbf{A}=\mathbf{0}$ in $\Omega$ and $\mathbf{E}_{C}=-i \omega\left(\mathbf{A}_{C}+\operatorname{grad} V_{C}\right)=\mathbf{0}$ in $\Omega_{C}$, and the uniqueness of the magnetic and electric fields is in any case verified.

In other words, the constraints $\mathcal{G}_{k}(\mathbf{A})=0$ seem not having any role in determining the right physical solution. This is true, but, since they are needed for well-posedness, they can have a role in the efficiency of the numerical algorithm used for approximation.

Indeed, as reported in Remark 6.1, it will be clear that for the finite element approximation well-posedness is satisfied even without imposing these constraints. However, the numerical computations presented in Section 7 are showing that in fact the efficiency of the numerical algorithm is better when the constraints are satisfied.

\section{Numerical approximation}

In this Section we present the finite element numerical approximation of problem (3.4). In the sequel we assume that $\Omega, \Omega_{C}$ and $\Omega_{I}$ are Lipschitz polyhedra, and that $\mathcal{T}_{I, h}$ and $\mathcal{T}_{C, h}$ are two regular families of triangulations of $\Omega_{I}$ and $\Omega_{C}$, respectively. For the sake of simplicity, we suppose that each element $K$ of $\mathcal{T}_{I, h}$ and $\mathcal{T}_{C, h}$ is a tetrahedron; however, the results below also hold for hexahedral elements (and for second order hexahedral "serendipity" elements). We also assume that these triangulations match on $\Gamma$, so that they furnish a family of triangulations $\mathcal{T}_{h}$ of $\Omega$.

Let $\mathbf{P}_{r}, r \geq 1$, be the space of polynomials of degree less than or equal to $r$. We will employ the discrete spaces given by nodal finite elements:

$$
\begin{aligned}
W_{h}^{r}:=\left\{\mathbf{v}_{h} \in\left(C^{0}(\bar{\Omega})\right)^{3} \mid \mathbf{v}_{h \mid K} \in\right. & \left(\mathbf{P}_{r}\right)^{3} \forall K \in \mathcal{T}_{h}, \mathbf{v}_{h} \cdot \mathbf{n}=0 \text { on } \partial \Omega \\
& \left.\mathcal{G}_{k}\left(\mathbf{v}_{h}\right)=0 \forall k=1, \ldots, n_{\partial \Omega}\right\}
\end{aligned}
$$

and

$$
\begin{aligned}
& X_{C, h}^{s}:=\left\{\phi_{C, h} \in C^{0}\left(\overline{\Omega_{C}}\right) \mid \phi_{C, h \mid K} \in \mathbf{P}_{s} \forall K \in \mathcal{T}_{C, h},\right. \\
&\left.\int_{\Omega_{C, j}} \phi_{C, h \mid \Omega_{C, j}}=0 \forall j=1, \ldots, p_{\Gamma}\right\} .
\end{aligned}
$$

Clearly, for each $r \geq 1$ and $s \geq 1$ we have $W_{h}^{r} \subset W$ and $X_{C, h}^{s} \subset H_{\sharp}^{1}\left(\Omega_{C}\right)$, therefore we are considering a conforming finite element approximation. 
As usual, the discrete problem is given by

$$
\begin{aligned}
& \text { find }\left(\mathbf{A}_{h}, V_{C, h}\right) \in W_{h}^{r} \times X_{C, h}^{s}: \\
& \mathcal{A}\left[\left(\mathbf{A}_{h}, V_{C, h}\right),\left(\mathbf{v}_{h}, \phi_{C, h}\right)\right]=\int_{\Omega} \mathbf{J}_{e} \cdot \overline{\mathbf{v}_{h}}+\int_{\Omega_{C}} \mathbf{J}_{e, C} \cdot \operatorname{grad} \overline{\phi_{C, h}} \\
& \forall\left(\mathbf{v}_{h}, \phi_{C, h}\right) \in W_{h}^{r} \times X_{C, h}^{s} . \\
& +\int_{\Gamma} \mathbf{J}_{e, I} \cdot \mathbf{n}_{I} \overline{\phi_{C, h}}
\end{aligned}
$$

Case 2 (or Case 1 with $\left.0=n_{\partial \Omega}=n_{\partial \Omega}^{*}\right)$.

Since the sesquilinear form $\mathcal{A}[\cdot, \cdot]$ is continuous and coercive, we have that the discrete solution exists and is unique; moreover, via Céa lemma for each $\mathbf{v}_{h} \in W_{h}^{r}$ and $\phi_{C, h} \in X_{C, h}^{s}$ we have

$$
\begin{aligned}
\kappa_{0}\left(\int _ { \Omega } \left(\left|\mathbf{A}-\mathbf{A}_{h}\right|^{2}+\right.\right. & \left.\left|\operatorname{rot}\left(\mathbf{A}-\mathbf{A}_{h}\right)\right|^{2}+\left|\operatorname{div}\left(\mathbf{A}-\mathbf{A}_{h}\right)\right|^{2}\right) \\
& \left.+\int_{\Omega_{C}}\left(\left|V_{C}-V_{C, h}\right|^{2}+\left|\operatorname{grad}\left(V_{C}-V_{C, h}\right)\right|^{2}\right)\right)^{1 / 2} \\
\leq C_{0}\left(\int_{\Omega}(\mid \mathbf{A}-\right. & \left.\left.\mathbf{v}_{h}\right|^{2}+\left|\operatorname{rot}\left(\mathbf{A}-\mathbf{v}_{h}\right)\right|^{2}+\left|\operatorname{div}\left(\mathbf{A}-\mathbf{v}_{h}\right)\right|^{2}\right) \\
& \left.+\int_{\Omega_{C}}\left(\left|V_{C}-\phi_{C, h}\right|^{2}+\left|\operatorname{grad}\left(V_{C}-\phi_{C, h}\right)\right|^{2}\right)\right)^{1 / 2}
\end{aligned}
$$

where $C_{0}>0$ is the continuity constant of $\mathcal{A}[\cdot, \cdot]$. Therefore, provided that the solutions $\mathbf{A}$ and $V_{C}$ are regular enough, by means of well-known interpolation results we find the error estimate

$$
\begin{aligned}
& \left(\int_{\Omega}\left(\left|\mathbf{A}-\mathbf{A}_{h}\right|^{2}+\left|\operatorname{rot}\left(\mathbf{A}-\mathbf{A}_{h}\right)\right|^{2}+\left|\operatorname{div}\left(\mathbf{A}-\mathbf{A}_{h}\right)\right|^{2}\right)\right. \\
& \left.\quad+\int_{\Omega_{C}}\left(\left|V_{C}-V_{C, h}\right|^{2}+\left|\operatorname{grad}\left(V_{C}-V_{C, h}\right)\right|^{2}\right)\right)^{1 / 2} \leq C h^{\min (r, s)} .
\end{aligned}
$$

(As it is known, the regularity of $\mathbf{A}$ and $V_{C}$ is not assured if $\Omega$ and $\Omega_{C}$ have reentrant corners, see Costabel and Dauge [10], Costabel, Dauge and Nicaise [11].)

Case 3 (or Case 1 with $0=n_{\partial \Omega}<n_{\partial \Omega}^{*}$ ).

In this case, we limit ourselves to the proof of the existence and uniqueness of the solution, without giving an error estimate. Since the problem is finite dimensional, the proof of uniqueness is enough.

Thus, let us consider a solution $\left(\mathbf{A}_{h}, V_{C, h}\right)$ to $(6.3)$ with a vanishing right hand side. As in the infinite dimensional case, from (5.3) we find that $\mathbf{A}_{h} \in$ $\mathcal{H}(m ; \Omega)$ and $\mathbf{A}_{h, C}+\operatorname{grad} V_{C, h}=\mathbf{0}$ in $\Omega_{C}$.

Since the harmonic fields in $\mathcal{H}(m ; \Omega)$ are $C^{\infty}$ vector functions in $\Omega$, we deduce that the piecewise polynomial $\mathbf{A}_{h}$ is indeed a global polynomial $\left(\mathbf{P}_{r}\right)^{3}$ in $\Omega$. Consequently, $\operatorname{rot} \mathbf{A}_{h}$ is a global polynomial $\left(\mathbf{P}_{r-1}\right)^{3}$ in $\Omega$, and there it is vanishing. Thus we have $\operatorname{rot} \mathbf{A}_{h}=\mathbf{0}$ in $\mathbf{R}^{3}$, and $\mathbf{A}_{h}=\operatorname{grad} U$ in $\mathbf{R}^{3}$. In particular, $\mathbf{A}_{h}=\operatorname{grad} U_{\mid \Omega}$ in $\Omega$, and the conditions $\operatorname{div} \mathbf{A}=0$ in $\Omega$ and $\mathbf{A}_{h} \cdot \mathbf{n}=0$ on $\partial \Omega$ tell us that $U_{\mid \Omega}$ is a harmonic function with vanishing normal derivative on the boundary, therefore is a constant. In conclusion, $\mathbf{A}_{h}=\mathbf{0}$ in $\Omega$ and therefore $V_{C, h}=0$ in $\Omega_{C}$. 
Remark 6.1. It is worth noting that at the discrete level, in all the geometrical cases 1, 2 and 3, one could consider the problem in the unconstrained space $\left(V \times H_{\sharp}^{1}\left(\Omega_{C}\right) \times\left(V \times H_{\sharp}^{1}\left(\Omega_{C}\right)\right.\right.$, where

$$
V:=\{\mathbf{v} \in H(\operatorname{rot} ; \Omega) \cap H(\operatorname{div} ; \Omega) \mid \mathbf{v} \cdot \mathbf{n}=0 \text { on } \partial \Omega\},
$$

still obtaining existence and uniqueness.

In fact, if the right hand side of the discrete equation is vanishing, from (5.3) one always finds $\mathbf{A}_{h} \in \mathcal{H}(m ; \Omega)$ and $\mathbf{A}_{h, C}+\operatorname{grad} V_{C, h}=\mathbf{0}$ in $\Omega_{C}$. Therefore, proceeding as before, one shows that $\mathbf{A}_{h}=\operatorname{grad} U_{\mid \Omega}$ in $\Omega$, and the uniqueness of the discrete solution again follows.

A natural question therefore arises: from the computational point of view, the constrained discrete approximation in the space $W$ is more efficient than the unconstrained one in the space $V$ ? One argument in favour of the constrained formulation is that, at least in the case $n_{\partial \Omega}=n_{\partial \Omega}^{*}$, we are able to prove an error estimate, therefore convergence is assured.

In the next Section we are going to present some numerical results that are confirming this assertion.

\section{Numerical results}

We consider the numerical approximation of problem (3.4) in Case 2, with $n_{\partial \Omega}=n_{\partial \Omega}^{*}=1$ ( $\Omega$ is a torus and $\Omega_{C}$ is a ball-like set).

We are using second order hexaedral "serendipity" elements, with 20 nodes ( 8 at the vertices and 12 at the midpoints of each edge), for all the components of $\mathbf{A}_{h}$ and for $V_{h}$.

The values of the physical coefficients have been assumed as follows: $\mu=$ $\mu_{*}=\mu_{0}=4 * \pi * 10^{-7} \mathrm{H} / \mathrm{m}, \sigma=5.7 * 10^{7} \mathrm{~S} / \mathrm{m}, \omega=2 * \pi * f=100 * \pi \mathrm{s}^{-1}$, i.e., $f=50 \mathrm{~Hz}$.

The CG iterations are stopped when the norm of the residual (normalized by the norm of the right hand side) is under a given tolerance. For the first two examples below, this tolerance is $10^{-10}$, while for the third example is $10^{-6}$.

In the first example, the (half of the) computational domain is described in Figure 1. The conductor $\Omega_{C}$ is green, the cutting surface $\Sigma_{1}$ is yellow and the coil (the support of $\mathbf{J}_{e, I}$ ) is red. We remark that all the results presented in this paper still hold true even if the basis of the eddy current domain $\Omega_{C}$ is touching the boundary $\partial \Omega$ as in Figure 1.

The difference between the constrained and the unconstrained finite element spaces resides only in one degree of freedom, the one associated to the "cut" $\Sigma_{1}$, cutting the equator of the torus $\Omega$. More precisely, in the constrained case we are assuming that trial and test functions satisfy $\int_{\Sigma_{1}} \mathbf{v}_{h} \cdot \mathbf{n}_{\Sigma}=0$. This can be done very easily; in fact, let us denote by $\phi_{i}$ the basis function of the unconstrained finite element space and set $c_{i}:=\int_{\Sigma_{1}} \phi_{i} \cdot \mathbf{n}_{\Sigma}$. If $c_{i}=0$ for each index $i$, there is nothing to do, as the unconstrained and the constrained space are coincident. Conversely, if for some index, say $i=1$, one has $c_{1} \neq 0$, for 
$i \geq 2$ define $\widehat{\phi}_{i}:=\phi_{i}-\frac{c_{i}}{c_{1}} \phi_{1}$. These functions are easily proved to be the basis functions of the constrained space.

The current density is given by $\mathbf{J}_{e, C}=\mathbf{0}$ and $\mathbf{J}_{e, I}=J_{e, I} \mathbf{e}_{\phi}$, where $\mathbf{e}_{\phi}$ is the azymuthal unit vector in the cylindrical system centered at the point $(100,0,0)$ (see Figure 1), and

$$
J_{e, I}= \begin{cases}10^{6} \mathrm{~A} / \mathrm{m}^{2} & \text { if } 60<r<80,60<z<80 \\ -10^{6} \mathrm{~A} / \mathrm{m}^{2} & \text { if } 60<r<80,20<z<40 \\ 0 & \text { otherwise }\end{cases}
$$

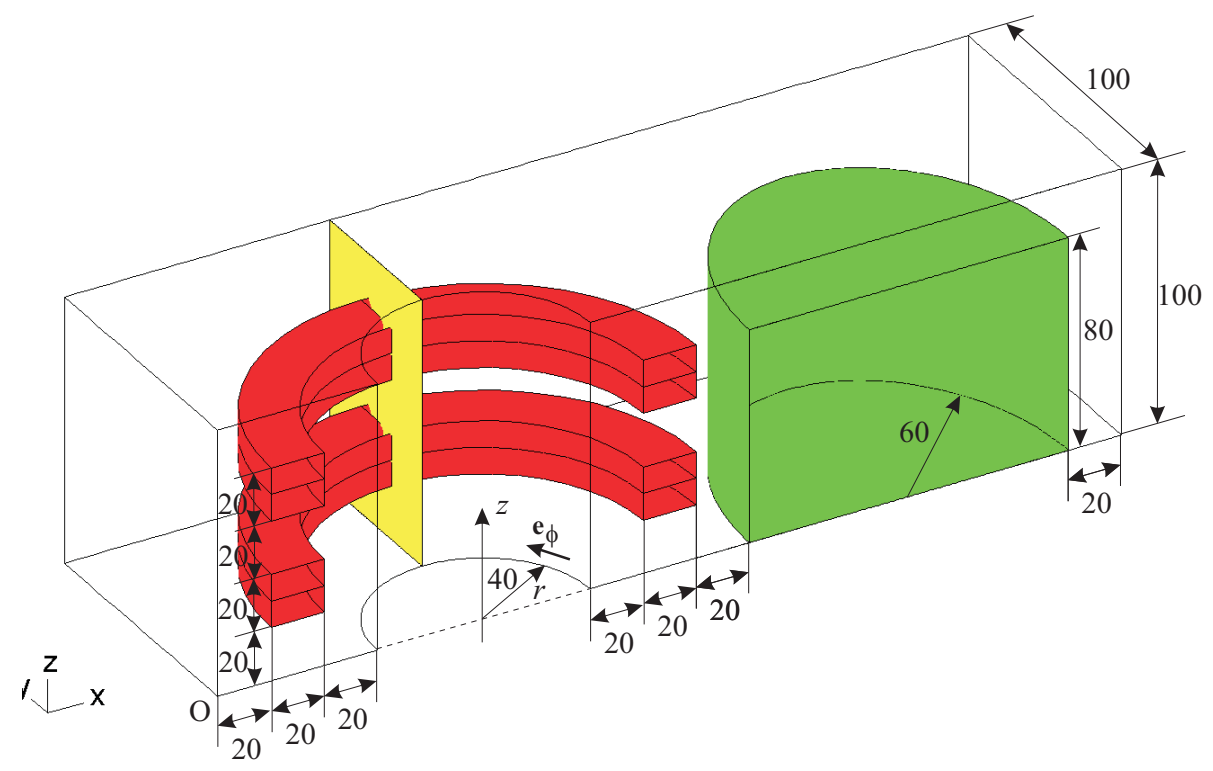

Figure 1: The first computational domain.

We have computed the solution for seven meshes, the coarsest one with 290 elements, the finest one with 99470 elements. With respect to the grid size, the seven meshes correspond to the choices $h, h / 2, \ldots, h / 7$.

For finding a reference solution, we have solved the problem by means of edge elements on the finest grid: this solution is called $\mathbf{A}_{\text {edge }}, V_{C \text {,edge }}$. For this computation, we have used the so-called quadratic 36-edge elements proposed by Kameari [18], writing the problem in terms of an ungauged magnetic vector potential and an electric scalar potential, namely, using the energy functional in (3.3) but dropping away the term containing the divergence (see also Bíró [5]).

In Table 1, for each of the meshes described above, we present the error between the computed solution $\mathbf{A}_{h}, V_{C, h}$ and the reference solution. More 
precisely, we set

$$
e_{\mathbf{J}}:=\frac{\sqrt{\int_{\Omega_{C}}\left|\mathbf{J}_{C, h}-\mathbf{J}_{C, \text { edge }}\right|^{2}}}{\sqrt{\int_{\Omega_{C}}\left|\mathbf{J}_{C, \text { edge }}\right|^{2}}}, \quad e_{\mathbf{B}}:=\frac{\sqrt{\int_{\Omega}\left|\mathbf{B}_{h}-\mathbf{B}_{\text {edge }}\right|^{2}}}{\sqrt{\int_{\Omega}\left|\mathbf{B}_{\text {edge }}\right|^{2}}},
$$

where $\mathbf{J}_{C \text {,edge }}:=-i \omega \sigma\left(\mathbf{A}_{C \text {,edge }}+\operatorname{grad} V_{C, \text { edge }}\right), \mathbf{B}_{\text {edge }}:=\operatorname{rot} \mathbf{A}_{\text {edge }}$, and similarly for $\mathbf{J}_{h}$ and $\mathbf{B}_{h}$. We also indicate the number of conjugate gradient iterations needed to compute the approximate solution. The computations are repeated twice, at first for the unconstrained approximate solution (namely, we have not imposed that the flux of the vector potential is vanishing on the cutting surface), and then for the constrained approximate solution. Clearly, in the latter case we have one degree of freedom less.

\begin{tabular}{|c|c||c|c|c|c|c|}
\hline Elements & DoF & Iterations & $e_{\mathbf{J}}$ & Rate & $e_{\mathbf{B}}$ & Rate \\
\hline \hline \multirow{2}{*}{290} & 3,939 & 108 & $1.60210^{-1}$ & - & $7.81210^{-2}$ & - \\
\cline { 2 - 7 } & 3,938 & 97 & $1.60210^{-1}$ & - & $7.81210^{-2}$ & - \\
\hline \multirow{2}{*}{2,320} & 31,337 & 206 & $5.63410^{-2}$ & 1.50 & $2.12510^{-2}$ & 1.88 \\
\cline { 2 - 7 } & 31,336 & 185 & $5.65910^{-2}$ & 1.50 & $2.12510^{-2}$ & 1.88 \\
\hline \multirow{2}{*}{7,830} & 105,571 & 325 & $2.78610^{-2}$ & 1.74 & $1.01510^{-2}$ & 1.82 \\
\cline { 2 - 7 } & 105,570 & 294 & $2.78310^{-2}$ & 1.74 & $1.01510^{-2}$ & 1.82 \\
\hline \multirow{2}{*}{18,560} & 250,017 & 448 & $1.60510^{-2}$ & 1.92 & $7.22810^{-3}$ & 1.16 \\
\cline { 2 - 7 } & 250,016 & 419 & $1.60210^{-2}$ & 1.92 & $7.22510^{-3}$ & 1.16 \\
\hline \multirow{2}{*}{36,250} & 488,051 & 597 & $1.05410^{-2}$ & 1.88 & $5.28610^{-3}$ & 1.40 \\
\cline { 2 - 7 } & 488,050 & 540 & $1.05210^{-2}$ & 1.88 & $5.28410^{-3}$ & 1.40 \\
\hline \multirow{2}{*}{62,640} & 843,049 & 739 & $7.60310^{-3}$ & 1.77 & $4.72910^{-3}$ & 0.61 \\
\cline { 2 - 7 } & 843,048 & 666 & $7.58810^{-3}$ & 1.78 & $4.72710^{-3}$ & 0.61 \\
\hline \multirow{2}{*}{99,470} & $1,338,387$ & 885 & $5.95910^{-3}$ & 1.58 & $4.22110^{-3}$ & 0.72 \\
\cline { 2 - 7 } & $1,338,386$ & 793 & $5.94810^{-3}$ & 1.58 & $4.21910^{-3}$ & 0.73 \\
\hline
\end{tabular}

Table 1: Relatives errors $e_{\mathbf{J}}$ and $e_{\mathbf{B}}$ for the first example.

It can be seen that the CG iterations are always approximately $10 \%$ fewer when computing the constrained solution, while the accuracy is quite similar in both cases. The rate of convergence is very often more than linear but it is less than quadratic (recall, however, that we are not comparing the approximate solutions with the exact solution, but with the edge element solution on the finest grid). Concerning the rate of convergence, more interesting results are presented in the other two examples below.

In Figures 2 and 3 we present some details of the computed solution for the finest mesh, and these pictures show a good agreement with the expected physical behaviour of the solution. 


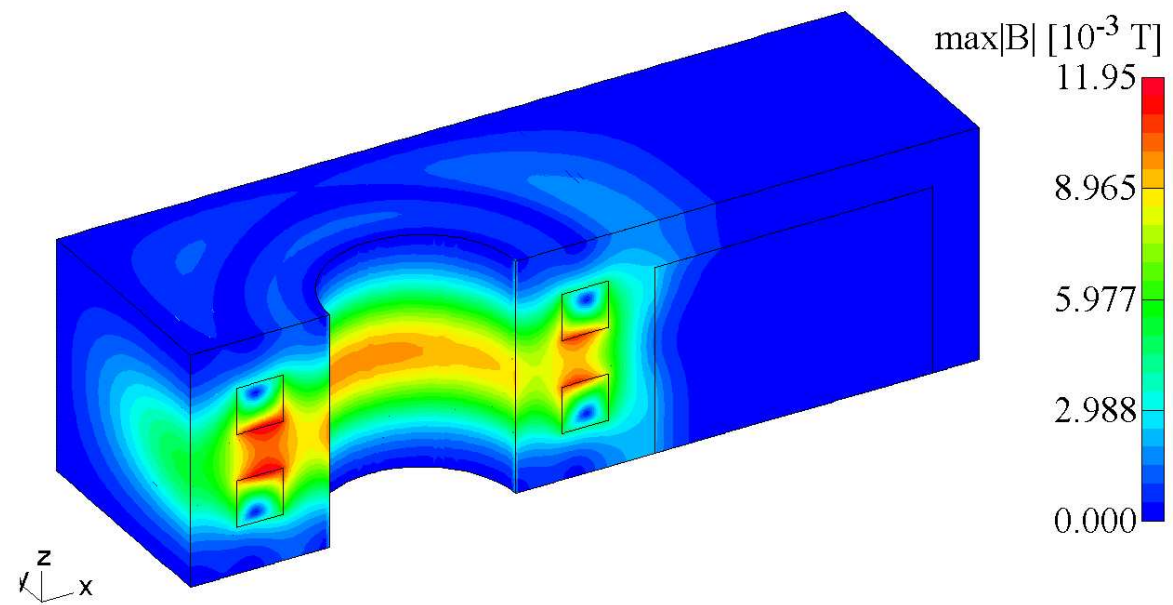

Figure 2: Magnitude of the computed flux density $\mathbf{B}$.

The second example is based on the smooth exact solution

$$
\begin{aligned}
& \mathbf{A}=\left\{\begin{array}{lr}
\operatorname{rot}\left(0,0, \exp \left(r^{2} / Q\right)\right) & \text { where } Q<0 \\
0 & \text { otherwise }
\end{array}\right. \\
& \operatorname{grad} V_{C}=\left\{\begin{array}{lr}
\operatorname{grad} \exp \left(r^{2} / Q\right) & \text { where } Q<0 \\
0 & \text { otherwise }
\end{array},\right.
\end{aligned}
$$

where

$$
Q:=\left(x-x_{0}\right)^{2}+\left(y-y_{0}\right)^{2}+\left(z-z_{0}\right)^{2}-r^{2},
$$

and $\left(x_{0}, y_{0}, z_{0}\right) \in \Omega, r>0$ can be chosen freely. Clearly, if we pose the ball $\{Q<0\}$ in $\Omega_{I}$, as we are going to do, we have $V_{C}=0$ and $\mathbf{E}_{C}=\mathbf{0}$. In particular, in this case the coil is the ball $\{Q<0\}$.

Considering the same domain $\Omega$ (and $\left.\Omega_{C}, \Omega_{I}\right)$ as before, we choose $\left(x_{0}, y_{0}, z_{0}\right)=$ $(60 / \sqrt{2}+100,60 / \sqrt{2}, 60)$ and $r=19$. In Table 2 we present the error between the computed solution $\mathbf{A}_{h}, V_{C, h}$ and the exact solution, setting

$$
\widehat{e}_{\mathbf{E}}:=\sqrt{\int_{\Omega_{C}}\left|\mathbf{E}_{C, h}\right|^{2}}, \quad e_{\mathbf{B}}:=\frac{\sqrt{\int_{\Omega}\left|\mathbf{B}_{h}-\mathbf{B}_{\text {exact }}\right|^{2}}}{\sqrt{\int_{\Omega}\left|\mathbf{B}_{\text {exact }}\right|^{2}}} .
$$

This time we start from a coarse mesh of size $h$ constituted by 150 elements, and then we take $h / 3, h / 5, h / 7$ and $h / 9$. The computations are repeated three 


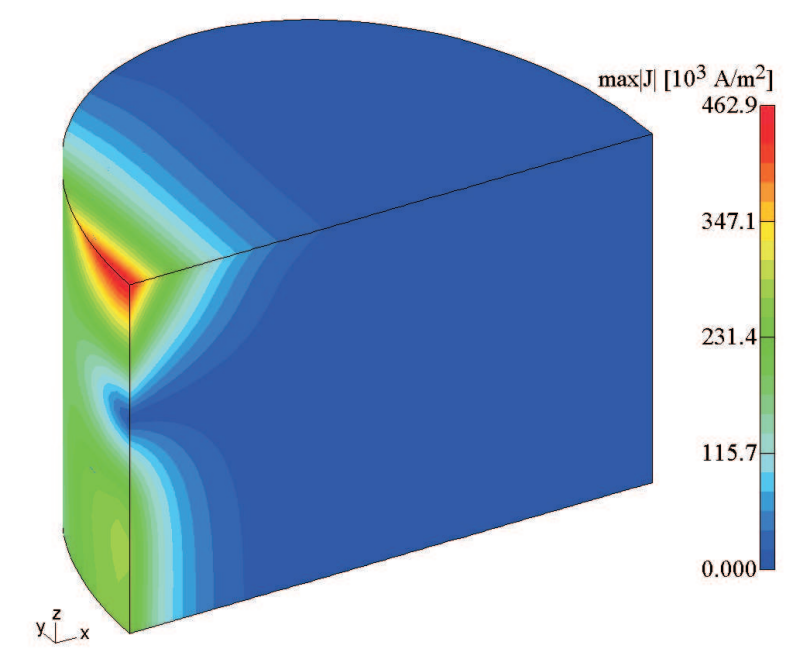

Figure 3: Magnitude of the computed current density $\mathbf{J}_{C}:=-i \omega \sigma\left(\mathbf{A}_{C}+\right.$ $\left.\operatorname{grad} V_{C}\right)$.

times: for the unconstrained algorithm, for the constrained algorithm, and for the edge element approximation. The results are reported in Table 2.

This second example shows again that the CG iterations for the constrained algorithm are less than in the other two cases; moreover, the accuracy of the constrained algorithm is much better than that of the unconstrained algorithm. In particular, when using the unconstrained approximation the absolute error for the electric field is not at all satisfactory even on the finest grid. Therefore, the advantage of the constrained algorithm is evident from this example.

The edge element approximation is the most accurate for what concerns the electric field, but is similar to that of the constrained algorithm for the magnetic field (in this case, however, both of them are still far from being satisfactory; this is due to the fact that the coil is quite small, and even on the finest mesh it is not represented in a good way).

The convergence is not always monotone with respect to $h$ (for some other computations we have verified even larger oscillations in the errors). However, it looks asymptotically quadratic for the electric field, though only linear for the magnetic field (whereas the theoretical estimate is quadratic, see (6.4)): in fact, passing from the coarsest to the finest mesh the global rate of convergence for the electric field is 1.54 for the unconstrained algorithm and 2.27 for the constrained one, while for the magnetic field it is 0.63 and 0.67 , respectively. In this respect, we note that a better order of convergence is achieved in the next example.

The third example is related to the exact solution of the form we described 


\begin{tabular}{|c|c||c|l|c|c|c|}
\hline Elements & DoF & Iterations & \multicolumn{1}{|c|}{$\widehat{e}_{\mathbf{E}}$} & Rate & $e_{\mathbf{B}}$ & Rate \\
\hline \hline \multirow{3}{*}{150} & 2,080 & 67 & $6.06110^{1}$ & - & $1.95510^{0}$ & - \\
\cline { 2 - 7 } & 2,079 & 59 & $7.48510^{0}$ & - & $1.68010^{0}$ & - \\
\cline { 2 - 7 } & 1,640 & 57 & $9,55610^{-2}$ & - & $9,89810^{-1}$ & - \\
\hline \multirow{3}{*}{1,050} & 55,192 & 199 & $1.43410^{2}$ & -0.78 & $3.10510^{0}$ & -0.42 \\
\cline { 2 - 7 } & 55,191 & 181 & $1.72710^{0}$ & 1.33 & $9.78410^{-1}$ & 0.49 \\
\cline { 2 - 7 } & 43,164 & 195 & $1,12310^{-2}$ & 1.95 & $7,75310^{-1}$ & 0.22 \\
\hline \multirow{3}{*}{51,450} & 254,536 & 357 & $1.02210^{1}$ & 5.16 & $7.89810^{-1}$ & 2.67 \\
\cline { 2 - 7 } & 254,535 & 319 & $3.89310^{-1}$ & 2.91 & $6.34310^{-1}$ & 0.84 \\
\cline { 2 - 7 } & 198,640 & 346 & $2,01410^{-3}$ & 3.36 & $6,12510^{-1}$ & 0.46 \\
\hline \multirow{3}{*}{109,350} & 697,264 & 553 & $3.48810^{0}$ & 3.19 & $5.74310^{-1}$ & 0.94 \\
\cline { 2 - 7 } & 697,263 & 459 & $1.84010^{-1}$ & 2.22 & $4.87010^{-1}$ & 0.78 \\
\cline { 2 - 7 } & 543,620 & 490 & $1,12110^{-3}$ & 1.73 & $4,78710^{-1}$ & 0.73 \\
\cline { 2 - 7 } & $1,480,528$ & 674 & $2.02210^{0}$ & 2.16 & $4.81510^{-1}$ & 0.70 \\
\cline { 2 - 7 } & $1,480,527$ & 591 & $5.05310^{-2}$ & 5.14 & $3.85610^{-1}$ & 0.93 \\
\hline
\end{tabular}

Table 2: Absolute error $\widehat{e}_{\mathbf{E}}$ and relative error $e_{\mathbf{B}}$ for the second example.

before, but for a different domain, described in Figures 4, 5, 6 .

As indicated in these figures, this time we choose $\left(x_{0}, y_{0}, z_{0}\right)=(0,0,0)$, $r=0.29$. The main difference with respect to the preceding situation is that now the coil is larger (and the eddy current region is smaller), so that the numerical approximation does not need very fine meshes for being satisfactory. We start from a coarse mesh of size $h$ constituted by 66 elements, and then we take $h / 2, h / 4, h / 8$ and $h / 16$. As before, the computations are repeated three times: for the unconstrained algorithm, for the constrained algorithm, and for the edge element approximation. The results are presented in Table 3.

In this last case, the accuracy of the unconstrained and constrained approximations is similar, and is good enough (however, the edge element approximation for the electric field is still the best one). The rate of convergence now looks to be asymptotically quadratic also for the magnetic field approximation.

\section{References}

[1] A. Alonso And A. VAlli, Some remarks on the characterization of the space of tangential traces of $H(\operatorname{rot} ; \Omega)$ and the construction of an extension operator, Manuscripta Mathematica, 89 (1996), pp. 159-178.

[2] — A domain decomposition approach for heterogeneous time-harmonic Maxwell equations, Comp. Meth. Appl. Mech. Engr., 143 (1997), pp. 97-112.

[3] A. Alonso Rodríguez, P. Fernandes, And A. VAlli, Weak and strong formulations for the time-harmonic eddy-current problem in general multi-connected domains, European J. Appl. Math., 14 (2003), pp. 387-406. 


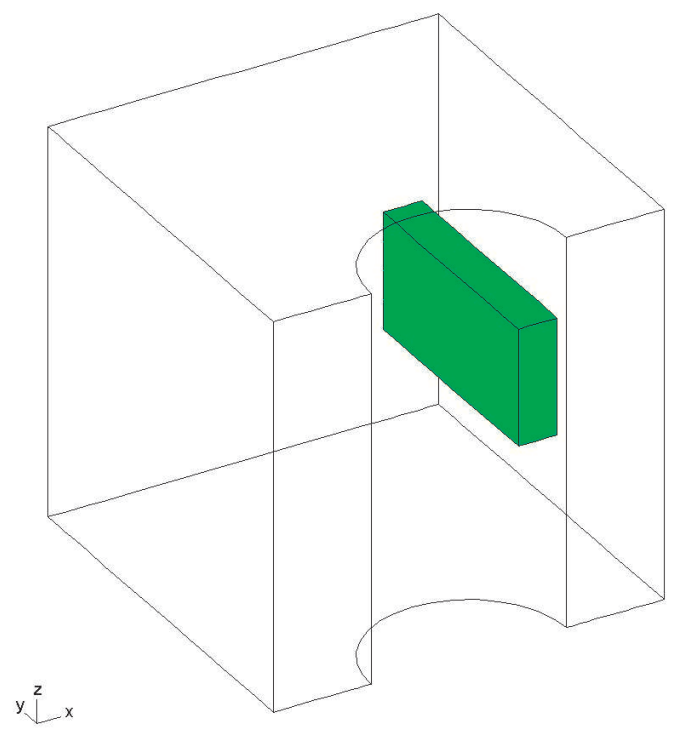

Figure 4: The second computational domain.

[4] C. Amrouche, C. Bernardi, M. Dauge, and V. Girault, Vector potentials in threedimensional nonsmooth domains, Math. Meth. Appl. Sci., 21 (1998), pp. 823-864.

[5] O. Bínó, Edge element formulation of eddy current problems, Comput. Methods Appl. Mech. Engrg., 169 (1999), pp. 391-405.

[6] O. Bíró AND K. Preis, On the use of magnetic vector potential in the finite element analysis of three-dimensional eddy currents, IEEE Trans. Magn., 25 (1989), pp. 31453159 .

[7] A. Bossavit, Computational Electromagnetism. Variational Formulation, Complementarity, Edge Elements, Academic Press, San Diego, 1998.

[8] — "Hybrid" electric-magnetic methods in eddy-current problems, Comput. Methods Appl. Mech. Eng., 178 (1999), pp. 383-391.

[9] - On the Lorenz gauge, COMPEL, 18 (1999), pp. 323-336.

[10] M. Costabel and M. Dauge, Singularities of electromagnetic fields in polyhedral domains, Arch. Ration. Mech. Anal., 151 (2000), pp. 221-276.

[11] M. Costabel, M. Dauge, and S. Nicaise, Singularities of eddy current problems, Math. Model. Numer. Anal., 37 (2003), pp. 807-831.

[12] R. Dautray and J.-L. Lions, Mathematical Analysis and NumericalMethods for Science and Technology. Volume 2: Functional and Variational Methods, Springer, Berlin, 1992.

[13] P. FERnAndes, General approach to prove the existence and uniqueness of the solution in vector potential formulations of 3-d eddy current problems, IEE Proc.-Sci. Meas. Technol., 142 (1995), pp. 299-306.

[14] P. Fernandes and G. Gilardi, Magnetostatic and electrostatic problems in inhomogeneous anisotropic media with irregular boundary and mixed boundary conditions, Math. Models Methods Appl. Sci., 7 (1997), pp. 957-991. 


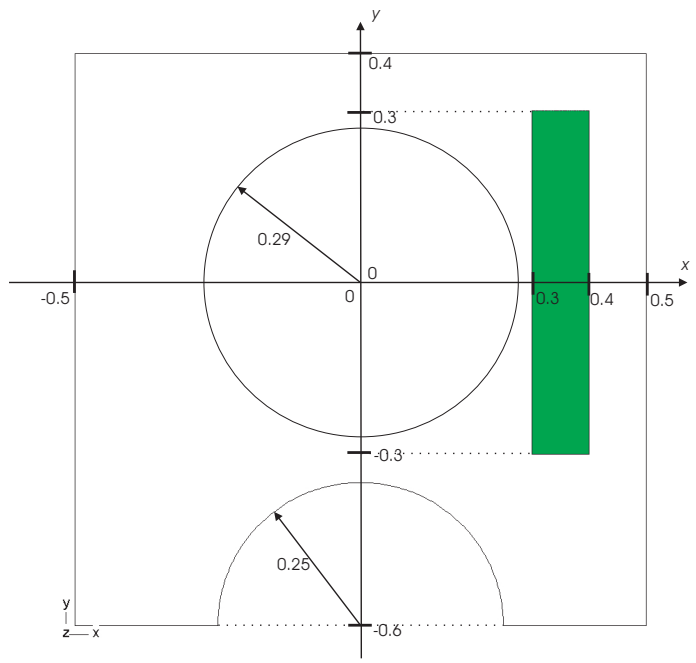

Figure 5: The second computational domain: projection on the $(x, y)$-plane.

[15] C. Foias And R. Temam, Remarques sur les équations de Navier-Stokes stationnaires et les phénomènes successifs de bifurcations, Ann. Scuola Norm. Sup. Pisa, 5 (IV) (1995), pp. 29-63.

[16] V. Girault and P. Raviart, Finite Element Methods for Navier-Stokes Equations, Springer, Berlin, 1986.

[17] R. Hiptmair, Finite elements in computational electromagnetism, Acta Numerica, 11 (2002), pp. 237-339.

[18] A. Kameari, Calculation of transient $3 d$ eddy current using edge-elements, IEEE Trans. Magn, 26 (1990), pp. 466-469.

[19] T. Morisue, Magnetic vector potential and electric scalar potential in three-dimensional eddy current problem, IEEE Trans. Magn., MAG-18 (1982), pp. 531-535.

[20] R. PiCARD, On the boundary value problems of electro- and magnetostatics, Proc. Royal Soc. Edinburgh, 92 A (1982), pp. 165-174.

[21] I. Tsukerman, Error estimation for finite element solutions of the eddy currents problem, COMPEL, 9 (1990), pp. 83-98.

Corresponding author:

Alberto Valli, Dipartimento di Matematica, Università di Trento, 38050

Povo (Trento), Italy

Tel: +390461 881580 Fax: +390461 881624

e-mail: valli@science.unitn.it 


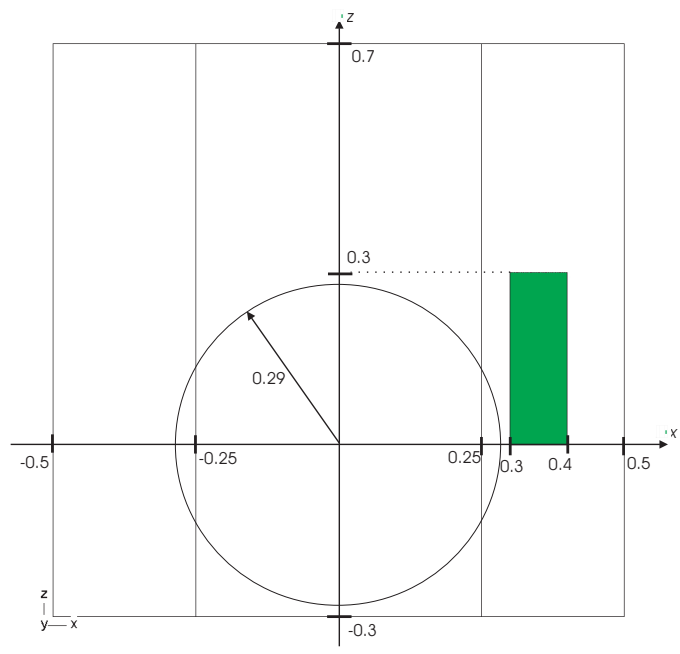

Figure 6: The second computational domain: projection on the $(x, z)$-plane.

\begin{tabular}{|c|c||c|c|c|c|c|}
\hline Elements & DoF & Iterations & $\widehat{e}_{\mathbf{E}}$ & Rate & $e_{\mathbf{B}}$ & Rate \\
\hline \hline \multirow{3}{*}{66} & 927 & 46 & $6.52410^{1}$ & - & $7.59510^{-1}$ & - \\
\cline { 2 - 7 } & 926 & 41 & $6.42410^{1}$ & - & $7,67610^{-1}$ & - \\
\cline { 2 - 7 } & 789 & 25 & $1,69710^{0}$ & - & $8,29810^{-1}$ & - \\
\hline \multirow{3}{*}{528} & 6,922 & 74 & $1,65410^{1}$ & 1.98 & $1,06410^{0}$ & -0.49 \\
\cline { 2 - 7 } & 6,921 & 63 & $1,07110^{1}$ & 2.58 & $9,37210^{-1}$ & -0.29 \\
\cline { 2 - 7 } & 5,539 & 62 & $1,10910^{0}$ & 0.61 & $7,70710^{-1}$ & 0.11 \\
\hline \multirow{3}{*}{33,224} & 53,322 & 135 & $7,18610^{0}$ & 1.20 & $6.48910^{-1}$ & 0.71 \\
\cline { 2 - 7 } & 53,321 & 117 & $6,64910^{0}$ & 0.69 & $6,20710^{-1}$ & 0.59 \\
\cline { 2 - 7 } & 41,373 & 137 & $4,38010^{-1}$ & 1.34 & $4,91010^{-1}$ & 0.65 \\
\hline \multirow{3}{*}{270,336} & 418,162 & 256 & $1,41510^{-1}$ & 5.67 & $2,22210^{-1}$ & 1.54 \\
\cline { 2 - 7 } & 418,161 & 227 & $1,41510^{-1}$ & 5.55 & $2,22210^{-1}$ & 1.48 \\
\cline { 2 - 7 } & 319,513 & 283 & $2,80310^{-2}$ & 3.97 & $2,50310^{-1}$ & 0.97 \\
\cline { 2 - 7 } & $3,311,202$ & 401 & $2,24410^{-2}$ & 2.66 & $6,88510^{-2}$ & 1.69 \\
\cline { 2 - 7 } & $2,311,201$ & 237 & $2,22510^{-2}$ & 2.67 & $6,88410^{-2}$ & 1.69 \\
\hline
\end{tabular}

Table 3: Absolute error $\widehat{e}_{\mathbf{E}}$ and relative error $e_{\mathbf{B}}$ for the third example. 